JOURNAL OF THE

AMERICAN MATHEMATICAL SOCIETY

Volume 24, Number 3, July 2011, Pages 871-898

S 0894-0347(2011)00694-2

Article electronically published on February 2, 2011

\title{
NON-COMMUTATIVE CIRCUITS AND THE SUM-OF-SQUARES PROBLEM
}

\author{
PAVEL HRUBEŠ, AVI WIGDERSON, AND AMIR YEHUDAYOFF
}

\section{INTRODUCTION}

1.1. Non-commutative computation. Arithmetic complexity theory studies the computation of formal polynomials over some field or ring. Most of this theory is concerned with the computation of commutative polynomials. The basic model of computation is that of an arithmetic circuit. Despite decades of work, the best size lower bound for general circuits computing an explicit $n$-variate polynomial of degree $d$ is $\Omega(n \log d)$, due to Baur and Strassen [32, 3. Better lower bounds are known for a variety of more restricted computational models, such as monotone circuits, multilinear or bounded depth circuits (see, e.g., 7, 4]).

In this paper we deal with a different type of restriction. We investigate noncommutative polynomials and circuits; the case when the variables do not multiplicatively commute, i.e., $x y \neq y x$ if $x \neq y$, as in the case when the variables represent matrices over a field 1 In a non-commutative circuit, a multiplication gate is given with an order in which its inputs are multiplied. Precise definitions appear in Section 2. A simple illustration of how absence of commutativity limits computation is the polynomial $x^{2}-y^{2}$. If $x, y$ commute, the polynomial can be computed as $(x-y)(x+y)$ using one multiplication. In the non-commutative case, two multiplications are required to compute it.

Surprisingly, while interest in non-commutative computations goes back at least to 1970 [36], no better lower bounds are known for general non-commutative circuits than in the commutative case. The seminal work in this area is 23, where Nisan proved exponential lower bounds on the non-commutative formula size of the determinant and the permanent. He also gives an explicit polynomial that has linear size non-commutative circuits but requires non-commutative formulas of exponential size, thus separating non-commutative formulas and circuits.

One remarkable aspect of non-commutative computation is its connection with the celebrated approximation scheme for the (commutative) permanent [16]. The series of papers [8, 18, 2, 6] reduce the problem of approximating the permanent to the problem of computing the determinant of a matrix whose entries are elements of (non-commutative) Clifford algebras. However, already in the case of quaternions (the third Clifford algebra), the determinant cannot be efficiently computed by means of arithmetic formulas. This was shown by Chien and Sinclair 5 who

Received by the editors April 30, 2010 and, in revised form, December 28, 2010.

2010 Mathematics Subject Classification. Primary 03D15, 68Q17; Secondary 11E25.

Key words and phrases. Algebraic complexity, composition of sums of squares.

The authors were supported in part by NSF Grant CCF 0832797.

${ }^{1}$ As in this case, addition remains commutative, as well as multiplication by constants.

(c)2011 American Mathematical Society 871

Reverts to public domain 28 years from publication 
extend Nisan's techniques to this and other non-commutative algebras. A recent work of Arvind and Srinivasan [1] shed new light on this approximation scheme. They showed that, in fact, if one is able to compute the determinant over the ring of matrices of polynomial size, then one can compute the permanent exactly, not just approximately.

In this paper, we propose new directions towards proving lower bounds on noncommutative circuits. We present structure theorems for non-commutative circuits, which enable us to reduce circuit-size lower bounds to apparently simpler problems. The foremost such problem is the so-called sum-of-squares problem, a classical question on a border between algebra and topology. We also outline a connection with multilinear circuits, in which exciting progress was made in recent years. We then make modest steps towards the lower-bound goal, and present results, some of which are of independent interest. Before we describe the results, we take a detour to briefly describe the sum-of-squares problem and its long history.

1.2. The sum-of-squares problem. In this section all variables commute. Consider the polynomial

$$
\operatorname{SOS}_{k}=\left(x_{1}^{2}+x_{2}^{2}+\cdots+x_{k}^{2}\right) \cdot\left(y_{1}^{2}+y_{2}^{2}+\cdots+y_{k}^{2}\right) .
$$

Given a field (or a ring) $\mathbb{F}$, define $\mathcal{S}_{\mathbb{F}}(k)$ as the smallest $n$ such that there exists a polynomial identity

$$
\mathrm{SOS}_{k}=z_{1}^{2}+z_{2}^{2}+\cdots+z_{n}^{2}
$$

where each $z_{i}=z_{i}(X, Y)$ is a bilinear form in the variables $X=\left\{x_{1}, \ldots, x_{k}\right\}$ and $Y=\left\{y_{1}, \ldots, y_{k}\right\}$ over the field $\mathbb{F}$.

We refer to the problem of determining the value $\mathcal{S}_{\mathbb{F}}(k)$ as the sum-of-squares problem. Note that the problem is not interesting if $\mathbb{F}$ has characteristic two, for then $\mathcal{S}_{\mathbb{F}}(k)=1$. Over other fields, the trivial bounds are

$$
k \leq \mathcal{S}_{\mathbb{F}}(k) \leq k^{2} .
$$

In Section 1.3, we describe the connection between the sum-of-squares problem and arithmetic complexity. At this point, let us discuss the mathematical significance of the sum-of-squares problem (much more can be found, e.g., in [31]). We focus on real sums of squares, for they are of the greatest historical importance 2 Non-trivial identities exhibiting $\mathcal{S}_{\mathbb{R}}(k)=k$ initiated this story.

When $k=1$, we have $x_{1}^{2} y_{1}^{2}=\left(x_{1} y_{1}\right)^{2}$. When $k=2$, we have

$$
\left(x_{1}^{2}+x_{2}^{2}\right) \cdot\left(y_{1}^{2}+y_{2}^{2}\right)=\left(x_{1} y_{1}-x_{2} y_{2}\right)^{2}+\left(x_{1} y_{2}+x_{2} y_{1}\right)^{2} .
$$

Interpreting $\left(x_{1}, x_{2}\right)$ and $\left(y_{1}, y_{2}\right)$ as complex numbers $\alpha$ and $\beta$, this formula expresses the property

$$
|\alpha|^{2}|\beta|^{2}=|\alpha \beta|^{2}
$$

of multiplication of complex numbers. The case $k=1$ trivially expresses the same fact (1.3) for real $\alpha$ and $\beta$. In 1748, motivated by the number-theoretic problem of expressing every integer as a sum of four squares, Euler proved an identity showing that $\mathcal{S}_{\mathbb{R}}(4)=4$. When Hamilton discovered the quaternion algebra in 1843 , this identity was quickly realized to express (1.3) for mutiplying quaternions. This was repeated in 1848 with the discovery of the octonions algebra, and the 8-square

\footnotetext{
${ }^{2}$ The assumption that the $z_{i}$ 's in $(1.2)$ are bilinear is satisfied automatically if the $z_{i}$ 's are real polynomials.
} 
identity expressing (1.3) for octonions. Motivated by the study of division algebras, mathematicians tried to prove a 16 -square identity in the following 50 years. Finally Hurwitz in 1898 proved that it is impossible, obtaining the first non-trivial lower bound:

Theorem $1.1([13])$. $\mathcal{S}_{\mathbb{R}}(k)>k$, except when $k \in\{1,2,4,8\}$.

The following interpretation of the sum-of-squares problem got topologists interested in this problem: if $z_{1}, \ldots, z_{n}$ satisfy (1.2), the map $z=\left(z_{1}, \ldots, z_{n}\right)$ : $\mathbb{R}^{k} \times \mathbb{R}^{k} \rightarrow \mathbb{R}^{n}$ is a bilinear normed map. Namely, it satisfies $|z(\bar{x}, \bar{y})|=|\bar{x}||\bar{y}|$ for every $\bar{x}, \bar{y} \in \mathbb{R}^{k}$, where $|\cdot|$ is the Euclidean norm. This rigid structure allows for topological and algebraic geometry tools to yield the following, best known lower bound, which unfortunately gains only a factor of two over the trivial bound:

Theorem $1.2([15,20]) . \mathcal{S}_{\mathbb{R}}(k) \geq(2-o(1)) k$.

As it happens, the trivial upper bound can be improved as well. There exists a normed bilinear map as above from $\mathbb{R}^{k} \times \mathbb{R}^{\rho(k)}$ to $\mathbb{R}^{k}$, with $\rho(k)=\Theta(\log k)$. This was shown by Radon and Hurwitz 26, 14, who computed the exact value of the optimal $\rho(k)$. Interestingly, such a map exists even if we require the polynomials $z_{i}$ to have integer 3 coefficients; see [39, 21. The existence of this integer bilinear normed map turns out to be related to Clifford algebras as well: it can be obtained using a matrix representation of a Clifford algebra with $\rho(k)$ generators. This can be seen to imply

Fact 1.3. $\mathcal{S}_{\mathbb{Z}}(k) \leq O\left(k^{2} / \log k\right)$.

This is the best known upper bound on $\mathcal{S}_{\mathbb{R}}$, or $\mathcal{S}_{\mathbb{F}}$ for any other field with char $\mathbb{F} \neq 2$. This motivated researchers to study integer sums of squares, and to try to prove lower bounds on $\mathcal{S}_{\mathbb{Z}}$. Despite the effort [20, 37, 31, the asymptotic bounds on $\mathcal{S}_{\mathbb{Z}}$ remained as wide open as in the case of reals. As a part of the project envisaged in this paper, the authors proved the first superlinear lower bound in the integer case:

Theorem $1.4([10]) . \mathcal{S}_{\mathbb{Z}}(k) \geq \Omega\left(k^{6 / 5}\right)$.

To illustrate the subtlety of proving lower bounds on the sum-of-squares problem, let us mention that if we allow the $z_{i}$ 's to be rational functions rather than polynomials, the nature of the problem significantly changes. In 1965, Pfister [25] proved that if the $z_{i}$ 's are rational functions, $\mathrm{SOS}_{k}$ can be written as a sum of $k$ squares whenever $k$ is a power of two.

\subsection{Non-commutative circuits and bilinear complexity.}

Conditional lower bounds on circuit complexity. The connection between the sumof-squares problem and non-commutative lower bounds is that a sufficiently strong lower bound on $\mathcal{S}(k)$ implies an exponential lower bound for the permanent. Here we present our main results; for a more detailed discussion, see Section 2.1. In the non-commutative setting, there are several options to define the permanent. We define it row-by-row, that is,

$$
\operatorname{PERM}_{n}(X)=\sum_{\pi} x_{1, \pi(1)} x_{2, \pi(2)} \cdots x_{n, \pi(n)},
$$

\footnotetext{
${ }^{3}$ The coefficients of the $z_{i}$ 's can actually be taken to be in $\{-1,0,1\}$.
} 
where $\pi$ is a permutation of $[n]=\{1, \ldots, n\}$. The advertised connection can be summarized as follows 4

Theorem 1.5. Let $\mathbb{F}$ be an algebraically closed field. Assume that $\mathcal{S}_{\mathbb{F}}(k) \geq \Omega\left(k^{1+\epsilon}\right)$ for a constant $\epsilon>0$. Then $\mathrm{PERM}_{n}$ requires non-commutative circuits of size $2^{\Omega(n)}$. In general, the non-commutative circuit-size of $\mathrm{PERM}_{4 n}$ is at least $\Omega\left(\frac{\mathcal{S}_{\mathbb{F}}\left(2^{n}\right)}{n^{3} 2^{n}}\right)$.

The theorem tells us that a non-trivial lower bound for the non-commutative circuit-size of the permanent holds even if $\mathcal{S}_{\mathbb{F}}(k)=\omega\left(k \log ^{5} k\right)$. We also note that any superlinear lower bound on $\mathcal{S}_{\mathbb{F}}(k)$ is interesting as it implies a superlinear lower bound for the non-commutative circuit-size of a constant degree polynomial (namely, the identity polynomial (1.4)).

Theorem 1.5 is an instance of a general connection between non-commutative circuits and commutative degree-four polynomials, which we now proceed to describe.

Let $f$ be a commutative polynomial of degree four over a field $\mathbb{F}$. We say that $f$ is biquadratic in variables $X=\left\{x_{1}, \ldots, x_{k}\right\}$ and $Y=\left\{y_{1}, \ldots, y_{k}\right\}$ if every monomial in $f$ has the form $x_{i_{1}} x_{i_{2}} y_{j_{1}} y_{j_{2}}$. If $f$ is biquadratic in variables $X$ and $Y$, we define

sum-of-squares complexity: $\mathcal{S}_{\mathbb{F}}(f)$ is the smallest $n$ so that $f$ can be written as

$$
f=z_{1}^{2}+\cdots+z_{n}^{2}
$$

bilinear complexity: $\mathcal{B}_{\mathbb{F}}(f)$ is the smallest $n$ so that $f$ can be written as

$$
f=z_{1} z_{1}^{\prime}+\cdots+z_{n} z_{n}^{\prime}
$$

where each $z_{i}$ and $z_{i}^{\prime}$ are bilinear forms in $X, Y$. We thus have $\mathcal{S}_{\mathbb{F}}\left(\operatorname{SOS}_{k}\right)=\mathcal{S}_{\mathbb{F}}(k)$, as defined in the previous section.

Let us first note that over certain fields, $\mathcal{S}_{\mathbb{F}}(f)$ and $\mathcal{B}_{\mathbb{F}}(f)$ are virtually the same:

Remark 1.6. Clearly, $\mathcal{B}_{\mathbb{F}}(f) \leq \mathcal{S}_{\mathbb{F}}(f)$. If $\mathbb{F}$ is algebraically closed with char $\mathbb{F} \neq 2$, then $\mathcal{S}_{\mathbb{F}}(f) \leq 2 \mathcal{B}_{\mathbb{F}}(f)$. This holds since $(1+1+1+1) z z^{\prime}=\left(z+z^{\prime}\right)^{2}+\left(\sqrt{-1}\left(z-z^{\prime}\right)\right)^{2}$.

We now define the non-commutative version of $\mathrm{SOS}_{k}$ : the non-commutative identity polynomial is

$$
\mathrm{ID}_{k}=\sum_{i, j \in[k]} x_{i} y_{j} x_{i} y_{j} .
$$

We show that a lower bound on $\mathcal{B}_{\mathbb{F}}\left(\mathrm{SOS}_{k}\right)$ implies a lower bound on the size of the non-commutative circuit computing $\mathrm{ID}_{k}$.

Theorem 1.7. The size of a non-commutative circuit over $\mathbb{F}$ computing $\mathrm{ID}_{k}$ is at least $\Omega\left(\mathcal{B}_{\mathbb{F}}\left(\mathrm{SOS}_{k}\right)\right)$.

Theorem 1.7 is proved in Section 4 The lower bound given by the theorem is reminiscent of the tensor rank approach to lower bounds for commutative circuits, where a lower bound on the tensor rank implies circuit lower bounds [33. In the non-commutative case we can prove a much stronger implication. For every $\epsilon>0$, a $k^{1+\epsilon}$ lower bound on $\mathcal{B}_{\mathbb{F}}\left(\mathrm{SOS}_{k}\right)$ gives an exponential lower bound for the permanent. Theorem 1.8, which is proved in Section 5, together with Remark 1.6 implies Theorem 1.5 .

\footnotetext{
${ }^{4}$ If char $\mathbb{F}=2$, the theorem holds trivially, since $\mathcal{S}_{\mathbb{F}}(k)=1$.

${ }^{5}$ When no such $n$ exists, $\mathcal{S}_{\mathbb{F}}(f)$ is infinite.
} 
Theorem 1.8. Assume that $\mathcal{B}_{\mathbb{F}}\left(\operatorname{SOS}_{k}\right) \geq \Omega\left(k^{1+\epsilon}\right)$ for some $\epsilon>0$. Then $\mathrm{PERM}_{n}$ requires non-commutative circuits of size $2^{\Omega(n)}$ over $\mathbb{F}$. In general, the non-commutative circuit-size of $\mathrm{PERM}_{4 n}$ is at least $\Omega\left(\frac{\mathcal{B}_{\mathbb{F}}\left(\mathrm{SOS}_{2 n}\right)}{n^{3} 2^{n}}\right)$.

The theorem is reminiscent of a result in Boolean complexity, where a sufficient linear lower bound on complexity of a bipartite graph implies an exponential circuit lower bound for a related function (see [17] for a discussion.)

An important property that the non-commutative permanent shares with its commutative counterpart is its completeness for the class of explicit polynomials. This enables us to generalize Theorem 1.8 to the following theorem, which is proved in Section 5.1. Let $\left\{f_{k}\right\}$ be a family of commutative biquadratic polynomials such that the number of variables in $f_{k}$ is polynomial in $k$. We call $\left\{f_{k}\right\}$ explicit if there exists a polynomial-time algorithm which, given $k$ and a degree-four monomial $\alpha$ as inputs 6 computes the coefficient of $\alpha$ in $f_{k}$. The polynomial $\mathrm{SOS}_{k}$ is clearly explicit.

Theorem 1.9. Let $\mathbb{F}$ be a field such that char $\mathbb{F} \neq 2$. Let $\left\{f_{k}\right\}$ be a family of explicit biquadratic polynomials. Assume that $\mathcal{B}_{\mathbb{F}}\left(f_{k}\right) \geq \Omega\left(k^{1+\epsilon}\right)$ for some $\epsilon>0$. Then $\mathrm{PERM}_{n}$ requires non-commutative circuits of size $2^{\Omega(n)}$ over $\mathbb{F}$.

Lower bounds on sum-of-squares complexity over real numbers. Remark 1.6 tells us that for some fields, $\mathcal{B}_{\mathbb{F}}=\Theta\left(\mathcal{S}_{\mathbb{F}}\right)$, and hence to prove a circuit lower bound, it is sufficient to prove a lower bound on $\mathcal{S}_{\mathbb{F}}$. We prove lower bounds on $\mathcal{S}_{\mathbb{F}}$ over reals.

Over $\mathbb{R}$, we find an explicit 'hard' polynomial (Theorem 1.10 is proved in Section 6).

Theorem 1.10. There exists an explicit family $\left\{f_{k}\right\}$ of real biquadratic polynomials with coefficients in $\{0,1,2,4\}$ such that $\mathcal{S}_{\mathbb{R}}\left(f_{k}\right)=\Theta\left(k^{2}\right)$.

By Theorem 1.9, if the construction worked over the complex numbers $\mathbb{C}$ instead of $\mathbb{R}$, we would have an exponential lower bound on the size of non-commutative circuits for the permanent. Such a construction is not known.

1.4. Ordered and multilinear circuits. An important restriction on computational power of circuits is multilinearity. This restriction has been extensively investigated in the commutative setting. A polynomial is multilinear if every variable has individual degree at most one in it. Syntactically multilinear circuits are those in which every product gate multiplies gates with disjoint sets of variables. This model was first considered in 24, where lower bounds on constant depth multilinear circuits were proved (and later improved in [29]). In a breakthrough paper, Raz [27] proved super-polynomial lower bounds on multilinear formula size for the permanent and determinant. These techniques were extended by [30] to give a lower bound of about $n^{4 / 3}$ for the size of the multilinear circuits.

An interesting observation about non-commutative circuits is that if they compute a polynomial of a specific form, they are without loss of generality multilinear. Let us call a non-commutative polynomial $f$ ordered, if the variables of $f$ are divided into disjoint sets $X_{1}, \ldots, X_{d}$ and every monomial in $f$ has the form $x_{1} \cdots x_{d}$ with $x_{i} \in X_{i}$. The non-commutative permanent, as defined above, is thus ordered. An ordered circuit is a natural model for computing ordered polynomials. Roughly, we

\footnotetext{
${ }^{6}$ We think of the input as given in a binary representation; the algorithm thus runs in time
} polynomial in $\log k$. 
require every gate to take variables from the sets $X_{i}$ in the same interval $I \subset[d]$; see Section 7.1 for a precise definition. One property of ordered circuits is that they are automatically syntactically multilinear. We remark that the notion of an ordered polynomial is defined when variables do not commute. It resembles the notion of a set-multilinear polynomial, which is defined when variables do commute.

We show that any non-commutative circuit computing an ordered polynomial can be efficiently transformed to an ordered circuit, hence a multilinear one, computing the same polynomial. Such a reduction is not known in the commutative case and gives hope that progress on multilinear lower bounds for the permanent or the determinant will yield general non-commutative lower bounds. Theorem 1.11 is proved in Section 7.1 .

Theorem 1.11. Let $f$ be an ordered polynomial of degree $d$. If $f$ is computed by a non-commutative circuit of size s, it can be computed by an ordered circuit of size $O\left(d^{3} s\right)$.

Again, we fall short of utilizing this connection for general lower bounds. By a simple argument, we manage to prove an exponential lower bound on noncommutative multilinear circuits, as we state in the next theorem. However, the polynomial $\mathrm{AP}_{k}$ in question is not ordered, and we cannot invoke the previous result to obtain an unconditional lower bound (Theorem 1.12 is proved in Section $[7.2$ ).

Theorem 1.12. Let

$$
\mathrm{AP}_{k}=\sum_{\sigma} x_{\sigma(1)} x_{\sigma(2)} \cdots x_{\sigma(k)},
$$

where $\sigma$ is a permutation of $[k]$. Then every non-commutative syntactically multilinear circuit computing $\mathrm{AP}_{k}$ is of size at least $2^{\Omega(k)}$.

1.5. A different perspective: lower bounds using rank. An extremely appealing way to obtain lower bounds is by using subadditive measures, and matrix rank is perhaps the favorite measure across many computational models.

For arithmetic circuits, many of the known structure theorems [9, 23, 27, 11] invite a natural rank interpretation. This interpretation, however, has lead to lower bounds only for restricted circuits. We sketch below the rank problem which arises in the case of commutative circuits and explain why it is considerably simpler in the case of non-commutative ones.

Let $f$ be a commutative polynomial of degree $d$. Consider $N \times N$ matrices whose entries are elements of some field, and rows and columns are labelled by monomials of degree roughly $d / 2$. Hence $N$ is in general exponential in the degree of $f$. Associate with $f$ a family $\mathcal{M}$ of all $N \times N$ matrices $M$ with the following property: for every monomial $\alpha$ of degree $d$, the sum of all entries $M_{\beta_{1}, \beta_{2}}$, such that $\beta_{1} \beta_{2}=\alpha$, is equal to the coefficient of $\alpha$ in $f$. In other words, we partition $M$ into subsets $T_{\alpha}$ corresponding to the possible ways to write $\alpha$ as a product of two monomials, and we impose a condition on the sum of entries in every $T_{\alpha}$. It can be shown that the circuit complexity of $f$ can be lower bounded by the minimal rank of the matrices in $\mathcal{M}$.

Note that the sets $T_{\alpha}$ are of size exponential in $d$, the degree of $f$. The structure of the sets is not friendly either. Our first structure theorem for non-commutative circuits, which decomposes non-commutative polynomials to central polynomials, translates to a similar rank problem. However, the matrices $M \in \mathcal{M}$ will be partitioned into sets of size only $d$ (instead of exponential in $d$ ). This is thanks to 
the fact that there are much fewer options to express a non-commutative monomial as a product of other monomials. Our second structure theorem, concerning blockcentral polynomials, gives a partition into sets of size at most two. The structure of these sets is quite simple too. However, it is not simple enough to allow us to prove a rank lower bound. In the rank formulation of circuit lower bounds, we can therefore see non-commutative circuits as a first step towards understanding commutative circuit lower bounds.

1.6. Structure of the paper. In Section 2 we outline our proofs of conditional lower bounds for non-commutative circuits and restricted lower bounds on the sumof-squares complexity. In Section 3 we investigate the structure of non-commutative circuits. In Section 4 we present a connection between circuit complexity of degreefour polynomials and bilinear complexity. In Section 5 we show a reduction from circuit complexity of general polynomials to bilinear complexity of degree-four polynomials; in particular, we prove a conditional lower bound for the permanent. In Section 6 we construct a polynomial whose sum-of-squares complexity over the reals in high. Finally, in Section 7 we study a family of circuits we call ordered, which are in particular multilinear, and prove a lower bound for the size of multilinear non-commutative circuits.

\section{OVERVIEW OF PROOFS}

2.1. Conditional lower bounds on non-commutative circuit-size. In this section we describe the path that leads from non-commutative circuit complexity to bilinear complexity.

Preliminaries. Let $\mathbb{F}$ be a field. A non-commutative polynomial is a formal sum of products of variables and field elements. We assume that the variables do not multiplicatively commute, that is, $x y \neq y x$ whenever $x \neq y$. However, the variables commute with elements of $\mathbb{F}$. The reader can imagine the variables as representing square matrices.

A non-commutative arithmetic circuit $\Phi$ is a directed acyclic graph as follows. Nodes (or gates) of in-degree zero are labelled by either a variable or a field element in $\mathbb{F}$. All the other nodes have in-degree two and they are labelled by either + or $\times$. The two edges going into a gate $v$ labelled by $\times$ are labelled by left and right. We denote by $v=v_{1} \times v_{2}$ the fact that $\left(v_{1}, v\right)$ is the left edge going into $v$, and $\left(v_{2}, v\right)$ is the right edge going into $v$. (This is to determine the order of multiplication.) The size of a circuit $\Phi$ is the number of edges in $\Phi$. The integer $\mathcal{C}(f)$ is the size of a smallest circuit computing $f$.

Remark 2.1. Unless stated otherwise, we refer to non-commutative polynomials as polynomials, and to non-commutative circuits as circuits.

The proof is presented in three parts, which are an exploration of the structure of non-commutative circuits.

Part I: Structure of circuits. The starting point of our trail is the structure of polynomials computed by non-commutative circuits, which we now explain. The methods we use are elementary and are an adaptation of works such as 9, 11] to the non-commutative world. 
We start by defining the 'building blocks' of polynomials, which we call central polynomials. A homogeneous 7 polynomial $f$ of degree $d$ is called central if there exist integers $m$ and $d_{0}, d_{1}, d_{2}$ satisfying $d / 3 \leq d_{0}<2 d / 3$ and $d_{0}+d_{1}+d_{2}=d$ so that

$$
f=\sum_{i \in[m]} h_{i} g \bar{h}_{i}
$$

where

(1) the polynomial $g$, which we call the body, is homogeneous of $\operatorname{degree} \operatorname{deg} g=$ $d_{0}$,

(2) for every $i \in[m]$, the polynomials $h_{i}, \bar{h}_{i}$ are homogeneous of degrees $\operatorname{deg} h_{i}$ $=d_{1}$ and $\operatorname{deg} \bar{h}_{i}=d_{2}$.

The width of a homogeneous polynomial $f$ of degree $d$, denoted $w(f)$, is the smallest integer $n$ so that $f$ can be written as

$$
f=f_{1}+f_{2}+\cdots+f_{n}
$$

with each $f_{i}$ a central polynomial. In Section 3.1 we show that the width of $f$ is at most $O\left(d^{3} \mathcal{C}(f)\right)$, and so lower bounds on width imply lower bounds on circuit complexity. We prove this by induction on the circuit complexity of $f$.

Part II: Degree four. In the first part, we argued that a lower bound on width implies a lower bound on circuit complexity. In the case of degree four, a central polynomial has a very simple structure: $d_{0}$ is always 2 , and so the body must reside in one of three places: left (when $d_{1}=0$ ), center (when $d_{1}=1$ ), and right (when $d_{1}=2$ ). For a polynomial of degree four, we can thus write (2.2) with $n$ at most order $\mathcal{C}(f)$, and each $f_{i}$ of this special form.

This observation allows us to relate width and bilinear complexity, as the following proposition shows. For a more general statement, see Proposition 4.1, which also shows that the width and bilinear complexity are in fact equivalent.

Proposition 2.2. $w\left(\mathrm{ID}_{k}\right) \geq \mathcal{B}\left(\mathrm{SOS}_{k}\right)$.

Part I and Proposition 2.2 already imply Theorem 1.7, which states that a lower bound on bilinear complexity implies a lower bound on circuit complexity of ID $_{k}$.

Part III: General degree to degree four. The argument presented in the second step can imply at most a quadratic lower bound on circuit-size. To get exponential lower bounds, we need to consider polynomials of higher degrees. We think of the degree of a degree- $4 r$ polynomial as divided into 4 groups, for which we try to mimic the special structure from part II: A block-central polynomial is a central polynomial so that $d_{0}=2 r$ and $d_{1} \in\{0, r, 2 r\}$. The structure of block-central polynomials is similar to the structure of degree-four central polynomials in that the body is of fixed degree and it has three places it can reside in: left (when $d_{1}=0$ ), center (when $d_{1}=r$ ), and right (when $d_{1}=2 r$ ). In Section 5 we show that a degree$4 r$ polynomial $f$ can be written as a sum of at most $O\left(r^{3} 2^{r} \mathcal{C}(f)\right)$ block-central polynomials.

We thus reduced the analysis of degree- $4 r$ polynomials to the analysis of degreefour polynomials. This reduction comes with a price, a loss of a factor of $2^{r}$. We

\footnotetext{
${ }^{7}$ Recall that a polynomial $f$ is homogeneous if all monomials with a non-zero coefficient in $f$ have the same degree, and that circuit $\Phi$ is homogeneous if every gate in $\Phi$ computes a homogeneous polynomial.
} 
note that this loss is necessary. The proof is a rather technical case distinction. The idea behind it is a combinatorial property of intervals in the set [4r], which allows us to transform a central polynomial to a sum of $2^{r}$ block-central polynomials.

Here is an example of this reduction in the case of the identity polynomial. The lifted identity polynomial, $\mathrm{LID}_{r}$, is the polynomial in variables $z_{0}, z_{1}$ of degree $4 r$ defined by

$$
\operatorname{LID}_{r}=\sum_{e \in\{0,1\}^{2 r}} z_{e} z_{e}
$$

where for $e=\left(e_{1}, \ldots, e_{2 r}\right) \in\{0,1\}^{2 r}$, we define $z_{e}=\prod_{i=1}^{2 r} z_{e_{i}}$. The lifted identity polynomial is the high-degree counterpart of the identity polynomial, which allows us to prove that a superlinear lower bound implies an exponential one (the corollary is proved in Section [5):

Corollary 2.3. If $\mathcal{B}\left(\mathrm{SOS}_{k}\right) \geq \Omega\left(k^{1+\epsilon}\right)$ for some $\epsilon>0$, then $\mathcal{C}\left(\mathrm{LID}_{r}\right) \geq 2^{\Omega(r)}$. In general, $\mathcal{C}\left(\mathrm{LID}_{r}\right) \geq \Omega\left(\frac{\mathcal{B}\left(\mathrm{SOS}_{2 r}\right)}{r^{3} 2^{r}}\right)$.

To complete the picture, we show that LID $_{r}$ is reducible to the permanent of dimension $4 r$.

Lemma 2.4. There exists a matrix $M$ of dimension $4 r \times 4 r$ whose non-zero entries are variables $z_{0}, z_{1}$ so that the permanent of $M$ is $\operatorname{LID}_{r}$.

To prove the lemma, the matrix $M$ is constructed explicitly; see Section 5 The conditional lower bound on the permanent, Theorem [1.8, follows from Corollary 2.3 and Lemma 2.4

An important property that the non-commutative permanent shares with its commutative counterpart is completeness for the class of explicit polynomials. This enables us to argue that a superlinear lower bound on the bilinear complexity of an explicit degree-four polynomial implies an exponential lower bound on the permanent. In the commutative setting, this is a consequence of the VNP completeness of the permanent, as given in [35]. In the non-commutative setting, one can prove a similar result [12]; see Section 5.1 for more details.

2.2. Lower bound on sum-of-squares over real numbers. We now discuss the lower bounds for the restricted sum-of-squares problem over the reals. For more details and formal definitions, see Section 6 .

We phrase the problem of lower bounding $\mathcal{S}_{\mathbb{R}}(g)$ in terms of matrices of real vectors. Let $V=\left\{\mathbf{v}_{i, j}: i, j \in[k]\right\}$ be a $k \times k$ matrix whose entries are vectors in $\mathbb{R}^{n}$. We call $V$ a vector matrix, and $n$ is called the height of $V$. The matrix $V$ defines a biquadratic polynomial $f(V)$ in $X=\left\{x_{1}, \ldots, x_{k}\right\}$ and $Y=\left\{y_{1}, \ldots, y_{k}\right\}$ by

$$
f(V)=\sum_{i_{1} \leq i_{2}, j_{1} \leq j_{2}} a_{i_{1}, i_{2}, j_{1}, j_{2}} x_{i_{1}} x_{i_{2}} y_{j_{1}} y_{j_{2}},
$$

where $a_{i_{1}, i_{2}, j_{1}, j_{2}}$ is equal to $\mathbf{v}_{i_{1}, j_{1}} \cdot \mathbf{v}_{i_{2}, j_{2}}+\mathbf{v}_{i_{1}, j_{2}} \cdot \mathbf{v}_{i_{2}, j_{1}}$, up to a small correction factor which is not important at this point (here, $\mathbf{v} \cdot \mathbf{v}^{\prime}$ is the inner product between the vectors $\left.\mathbf{v}, \mathbf{v}^{\prime}\right)$. We can think of the coefficients as given by the permanent of the $2 \times 2$ submatrix 8 of $V$ defined by $i_{1}, i_{2}$ and $j_{1}, j_{2}$.

\footnotetext{
${ }^{8}$ In some cases, e.g., when $i_{1}=i_{2}$, this matrix can become $1 \times 2,2 \times 1$ or even $1 \times 1$, but we still think of it as a $2 \times 2$ matrix. This is also where the correction factor comes from.
} 
The following lemma, whose version is proved in Section 6, gives the connection between sum-of-squares complexity and vector matrices.

Lemma 2.5. Let $g$ be a biquadratic polynomial. Then $\mathcal{S}_{\mathbb{R}}(g) \leq n$ is equivalent to the existence a vector matrix $V$ of height $n$ so that $g=f(V)$.

As long as it is finite, the height of a vector matrix for any polynomial does not exceed $k^{2}$, and a counting argument shows that "almost" all polynomials require height $\Omega\left(k^{2}\right)$. The problem is to construct explicit polynomials that require a large height. Even a superlinear lower bound seems non-trivial, since the permanent condition does not talk about inner products of pairs of vectors, but rather about the sum of inner products of two such pairs. In Section 6 we manage to construct an explicit polynomial which requires near-maximal height $\Omega\left(k^{2}\right)$. In our proof, the coefficients impose (through the $2 \times 2$ permanent conditions) either equality or orthognality constraints on the vectors in the matrix, and eventually the existence of many pairwise orthogonal ones. In a crucial way, we employ the fact that over $\mathbb{R}$, if two unit vectors have inner product one, they must be equal. This property 9 fails over $\mathbb{C}$, but it is still possible that even over $\mathbb{C}$ our construction has a similar height (of course, if this turns out to be even $k^{1+\epsilon}$, we get an exponential lower bound for non-commutative circuits).

The construction, however, does not shed light on the classical sum-of-squares problem which is concerned specifically with the polynomial $\mathrm{SOS}_{k}$. However, note that Lemma 2.5 gives a potentially useful formulation of the sum-of-squares problem. In the case of $\mathrm{SOS}_{k}$, the conditions on the matrix $V$ are especially nice and simple: (1) all vectors in $V$ are unit vectors, (2) in each row and column the vectors are pairwise orthogonal, and (3) every $2 \times 2$ permanent (of inner products) must be zero. Indeed, this formulation was used in [10] by the authors to prove a lower bound on integer sum-of-squares.

\section{Non-commutative circuits}

We use the following notation. For a node $v$ in a circuit $\Phi$, we denote by $\Phi_{v}$ the subcircuit of $\Phi$ rooted at $v$. Every node $v$ computes a polynomial $\widehat{\Phi}_{v}$ in the obvious way. A monomial $\alpha$ is a product of variables, and $\operatorname{COEF}_{\alpha}(f)$ is the coefficient of $\alpha$ in the polynomial $f$. Denote by $\operatorname{deg} f$ the degree of $f$, and if $v$ is a node in a circuit $\Phi$, denote by $\operatorname{deg} v$ the degree of $\widehat{\Phi}_{v}$.

3.1. Structure of non-commutative circuits. In this section we describe the structure of the polynomials computed by non-commutative circuits. The methods we use are elementary and are an adaptation of works such as [9, 11] to the noncommutative world.

We start by defining the 'building blocks' of polynomials, which we call central polynomials. Recall that a polynomial $f$ is homogeneous if all monomials with a non-zero coefficient in $f$ have the same degree, and that a circuit $\Phi$ is homogeneous if every gate in $\Phi$ computes a homogeneous polynomial. A homogeneous polynomial $f$ of degree $d$ is called central if there exist integers $m$ and $d_{0}, d_{1}, d_{2}$ satisfying

$$
d / 3 \leq d_{0}<2 d / 3 \text { and } d_{0}+d_{1}+d_{2}=d
$$

\footnotetext{
${ }^{9}$ Here, the inner product of two complex vectors $a, b$ is $\sum_{i} a_{i} b_{i}$, rather than $\sum_{i} a_{i} \bar{b}_{i}$, with $\bar{b}$ the complex conjugate of $b$.
} 
so that

$$
f=\sum_{i \in[m]} h_{i} g \bar{h}_{i},
$$

where

(1) the polynomial $g$ is homogeneous of degree $\operatorname{deg} g=d_{0}$,

(2) for every $i \in[m]$, the polynomials $h_{i}, \bar{h}_{i}$ are homogeneous of degrees $\operatorname{deg} h_{i}$ $=d_{1}$ and $\operatorname{deg} \bar{h}_{i}=d_{2}$.

Remark 3.1. In the definition of a central polynomial, no assumption on the size of $m$ is made. Hence we can without loss of generality assume that $h_{i}=c_{i} \alpha_{i}$ and $\bar{h}_{i}=\beta_{i}$, where $\alpha_{i}$ is a monomial of degree $d_{1}, \beta_{i}$ is a monomial of degree $d_{2}$, and $c_{i}$ is a field element.

The width of a homogeneous polynomial $f$ of degree $d$, denoted $w(f)$, is the smallest integer $n$ so that $f$ can be written as

$$
f=f_{1}+f_{2}+\cdots+f_{n},
$$

where $f_{1}, \ldots, f_{n}$ are central polynomials of degree $d$. The following proposition shows that the width of a polynomial is a lower bound for its circuit complexity. We will later relate width and bilinear complexity.

Proposition 3.2. Let $f$ be a homogeneous polynomial of degree $d \geq 2$. Then

$$
\mathcal{C}(f) \geq \Omega\left(d^{-3} w(f)\right) .
$$

Proof. We start by observing that the standard homogenization of commutative circuits [33, 4] works for non-commutative circuits as well (and we omit the proof).

Lemma 3.3. Let $g$ be a homogeneous polynomial of degree $d$. Then there exists a homogeneous circuit of size $O\left(d^{2} \mathcal{C}(f)\right)$ computing $g$.

Assume that we have a homogeneous circuit $\Phi$ of size $s$ computing $f$. We will show that $w(f) \leq d s$. By Lemma 3.3, this implies that $w(f) \leq O\left(d^{3} \mathcal{C}(f)\right)$, which completes the proof. Without loss of generality, we can also assume that no gate $v$ in $\Phi$ computes the zero polynomial (gates that compute the zero polynomial can be removed, decreasing the circuit-size).

For a multiset of pairs of polynomials $\mathcal{H}=\left\{\left\langle h_{i}, \bar{h}_{i}\right\rangle: i \in[m]\right\}$, define

$$
g \times \mathcal{H}=\sum_{i \in[m]} h_{i} g \bar{h}_{i} .
$$

Let $\mathcal{G}=\left\{g_{1}, \ldots, g_{t}\right\}$ be the set of homogeneous polynomials $g$ of degree $d / 3 \leq$ $\operatorname{deg} g<2 d / 3$ so that there exists a gate in $\Phi$ computing $g$. We show that for every gate $v$ in $\Phi$ so that $\operatorname{deg} v \geq d / 3$ there exist multisets of pairs of homogeneous polynomials $\mathcal{H}_{1}(v), \ldots, \mathcal{H}_{t}(v)$ satisfying

$$
\widehat{\Phi}_{v}=\sum_{i \in[t]} g_{i} \times \mathcal{H}_{i}(v) .
$$

We prove (3.2) by induction on the depth of $\Phi_{v}$. If $\operatorname{deg}(v)<2 d / 3$, then $\widehat{\Phi}_{v}=g_{i} \in \mathcal{G}$ for some $i \in[t]$. Thus (3.2) is true, setting $\mathcal{H}_{i}(v)=\{\langle 1,1\rangle\}$ and $\mathcal{H}_{j}(v)=\{\langle 0,0\rangle\}$ for $j \neq i$ in $[t]$. Otherwise, we have $\operatorname{deg} v \geq 2 d / 3$. When $v=v_{1}+v_{2}$, we do the 
following. Since $\Phi$ is homogeneous, $v_{1}, v_{2}$ and $v$ have the same degree, which is at least $2 d / 3$. Induction thus implies: for every $e \in\{1,2\}$,

$$
\widehat{\Phi}_{v_{e}}=\sum_{i \in[t]} g_{i} \times \mathcal{H}_{i}\left(v_{e}\right)
$$

This gives

$$
\widehat{\Phi}_{v}=\widehat{\Phi}_{v_{1}}+\widehat{\Phi}_{v_{2}}=\sum_{i \in[t]} g_{i} \times\left(\mathcal{H}_{i}\left(v_{1}\right) \cup \mathcal{H}_{i}\left(v_{2}\right)\right) .
$$

When $v=v_{1} \times v_{2}$, we have $\operatorname{deg} v=\operatorname{deg} v_{1}+\operatorname{deg} v_{2}$. Since $\operatorname{deg} v \geq 2 d / 3$, either (a) $\operatorname{deg} v_{1} \geq d / 3$ or $(\mathrm{b}) \operatorname{deg} v_{2} \geq d / 3$. In the case (a), by induction,

$$
\widehat{\Phi}_{v_{1}}=\sum_{i \in[t]} g_{i} \times \mathcal{H}_{i}\left(v_{1}\right) \text {. }
$$

Defining $\mathcal{H}_{i}(v)=\left\{\left\langle h, \bar{h} \widehat{\Phi}_{v_{2}}\right\rangle:\langle h, \bar{h}\rangle \in \mathcal{H}_{i}\left(v_{1}\right)\right\}$, we obtain

$$
\widehat{\Phi}_{v}=\widehat{\Phi}_{v_{1}} \widehat{\Phi}_{v_{2}}=\left(\sum_{i \in[t]} g_{i} \times \mathcal{H}_{i}\left(v_{1}\right)\right) \widehat{\Phi}_{v_{2}}=\sum_{i \in[t]} g_{i} \times \mathcal{H}_{i}(v) .
$$

Since $\widehat{\Phi}_{v_{2}}$ is a homogeneous polynomial, $\mathcal{H}_{i}(v)$ consists of pairs of homogeneous polynomials. In case $(\mathrm{b})$, define $\mathcal{H}_{i}(v)=\left\{\left\langle\widehat{\Phi}_{v_{1}} h, \bar{h}\right\rangle:\langle h, \bar{h}\rangle \in \mathcal{H}_{i}\left(v_{2}\right)\right\}$.

Applying (3.2) to the output gate of $\Phi$, we obtain

$$
f=\sum_{i \in[t]} g_{i} \times \mathcal{H}_{i}
$$

where $\mathcal{H}_{i}$ are multisets of pairs of homogeneous polynomials. For every $i \in[t]$ and every $r \leq d-\operatorname{deg} g_{i}$, define $\mathcal{H}_{i}^{r}=\left\{\langle h, \bar{h}\rangle \in \mathcal{H}_{i}: \operatorname{deg}(h)=r, \operatorname{deg} \bar{h}=d-\operatorname{deg} g_{i}-r\right\}$. Then $g_{i} \times \mathcal{H}_{i}^{r}$ is a central polynomial. Moreover, since $f$ is homogeneous of degree $d$, we obtain

$$
f=\sum_{i \in[t]} \sum_{r=0}^{d-\operatorname{deg} g_{i}} g_{i} \times \mathcal{H}_{i}^{r}
$$

Since $t \leq s$, the proof is complete.

3.2. Degree-four polynomials. Before we describe the specific structure of degreefour polynomials, let us give general definitions. For a monomial $\alpha$ and a variable $x$, we say that $x$ occurs at position $i$ in $\alpha$, if $\alpha=\alpha_{1} x \alpha_{2}$ and $\operatorname{deg} \alpha_{1}=i-1$. Let $X_{1}, \ldots, X_{r}$ be (not necessarily disjoint) sets of variables. For a polynomial $f$, let $f\left[X_{1}, \ldots, X_{r}\right]$ be the homogeneous polynomial of degree $r$ so that for every monomial $\alpha$,

$$
\operatorname{COEF}_{\alpha}\left(f\left[X_{1}, \ldots, X_{r}\right]\right)= \begin{cases}\operatorname{COEF}_{\alpha}(f) & \text { if } \alpha=x_{1} x_{2} \cdots x_{r} \\ & \text { with } x_{i} \in X_{i} \text { for every } i \in[r] \\ 0 & \text { otherwise. }\end{cases}
$$

In other words, $f\left[X_{1}, \ldots, X_{r}\right]$ is the part of $f$ consisting of monomials of degree $r$ with the property that if a variable $x$ occurs at a position $i$, then $x \in X_{i}$. 
Claim 3.4. Let $f$ be a central polynomial so that $f=f\left[X_{1}, X_{2}, X_{3}, X_{4}\right]$. Then, either

$$
f=g\left[X_{1}, X_{2}\right] h\left[X_{3}, X_{4}\right] \quad \text { or } \quad f=\sum_{i \in[m]} h_{i}\left[X_{1}\right] g\left[X_{2}, X_{3}\right] \bar{h}_{i}\left[X_{4}\right],
$$

where $g, h, h_{i}, \bar{h}_{i}$ are some polynomials.

Proof. As $f$ is central of degree four, $\operatorname{deg} g=d_{0}=2$, and $d_{1} \in\{0,1,2\}$. If $d_{1}=1$, then $d_{2}=1$ as well, and

$f=f\left[X_{1}, X_{2}, X_{3}, X_{4}\right]=\sum_{i \in[m]}\left(h_{i} g \bar{h}_{i}\right)\left[X_{1}, X_{2}, X_{3}, X_{4}\right]=\sum_{i \in[m]} h_{i}\left[X_{1}\right] g\left[X_{2}, X_{3}\right] \bar{h}_{i}\left[X_{4}\right]$.

If $d_{1}=0$, then $d_{2}=2$, and

$f=f\left[X_{1}, X_{2}, X_{3}, X_{4}\right]=\sum_{i \in[m]}\left(g \bar{h}_{i}\right)\left[X_{1}, X_{2}, X_{3}, X_{4}\right]=g\left[X_{1}, X_{2}\right]\left(\sum_{i \in[m]} h_{i}\left[X_{1}\right] \bar{h}_{i}\left[X_{4}\right]\right)$.

A similar argument holds when $d_{1}=2$.

Claim 3.4 implies the following lemma.

Lemma 3.5. If $f=f\left[X_{1}, X_{2}, X_{3}, X_{4}\right]$, then $w(f)$ is the smallest $n$ so that $f$ can be written as $f=f_{1}+\cdots+f_{n}$, where for every $t \in[n]$, either

(a) $f_{t}=g_{t}\left[X_{1}, X_{2}\right] h_{t}\left[X_{3}, X_{4}\right]$, or

(b) $f_{t}=\sum_{i \in[m]} h_{t, i}\left[X_{1}\right] g_{t}\left[X_{2}, X_{3}\right] \bar{h}_{t, i}\left[X_{4}\right]$,

where $g_{t}, h_{t}, h_{t, i}, \bar{h}_{t, i}$ are some polynomials.

\section{Degree four and Bilinear COMPleXity}

We consider polynomials of a certain structure. Let $f$ be a polynomial in variables $X=\left\{x_{1}, \ldots, x_{k}\right\}$ and $Y=\left\{y_{1}, \ldots, y_{k}\right\}$ so that $f=f[X, Y, X, Y]$, i.e.,

$$
f=\sum_{i_{1}, j_{1}, i_{2}, j_{2} \in[k]} a_{i_{1}, j_{1}, i_{2}, j_{2}} x_{i_{1}} y_{j_{1}} x_{i_{2}} y_{j_{2}} .
$$

For a non-commutative polynomial $g$, we define $g^{(c)}$ to be the polynomial $g$ understood as a commutative polynomial. For example, if $g=x y+y x$, then $g^{(c)}=2 x y$. In particular, if $f$ is of the form (4.1), the polynomial $f^{(c)}$ is biquadratic. We say that $f$ is $(X, Y)$-symmetric if for every $i_{1}, j_{1}, i_{2}, j_{2} \in[k]$,

$$
a_{i_{1}, j_{1}, i_{2}, j_{2}}=a_{i_{2}, j_{1}, i_{1}, j_{2}}=a_{i_{1}, j_{2}, i_{2}, j_{1}}=a_{i_{2}, j_{2}, i_{1}, j_{1}} .
$$

In the following proposition, we relate the width of a polynomial $f$ and $\mathcal{B}\left(f^{(c)}\right)$.

Proposition 4.1. Let $f$ be a homogeneous polynomial of degree four of the form (4.1). Then

(1) $\mathcal{B}\left(f^{(c)}\right) \leq w(f)$, and

(2) if char $\mathbb{F} \neq 2$ and $f$ is $(X, Y)$-symmetric, then $w(f) \leq 4 \mathcal{B}\left(f^{(c)}\right)$. 
Proof. We start by proving (11). Using Lemma 3.5, we can write $f=f_{1}+\cdots+f_{n}$, where for every $t \in[n]$, either

(a) $f_{t}=g_{t}[X, Y] h_{t}[X, Y]$, or

(b) $f_{t}=\sum_{i \in[m]} h_{t, i}[X] g_{t}[Y, X] \bar{h}_{t, i}[Y]$.

The commutative polynomial $f_{t}^{(c)}$ is a product of two bilinear forms in $X$ and $Y$ : in case (a), of $g_{t}[X, Y]^{(c)}$ and $h_{t}[X, Y]^{(c)}$, and in case (b), of $g_{t}[Y, X]^{(c)}$ and $\sum_{i \in[m]} h_{t, i}[X]^{(c)} \bar{h}_{t, i}[Y]^{(c)}$. Altogether $f^{(c)}=f_{1}^{(c)}+\cdots+f_{n}^{(c)}$, where each $f_{t}^{(c)}$ is a product of two bilinear forms, and hence $\mathcal{B}\left(f^{(c)}\right) \leq n$.

We now prove (2). Assume that

$$
f^{(c)}=z_{1} z_{1}^{\prime}+\cdots+z_{n} z_{n}^{\prime}
$$

where $z_{t}$ and $z_{t}^{\prime}, t \in[n]$, are bilinear in $X$ and $Y$. Write

$$
z_{t}=\sum_{j \in[k]} x_{j} g_{t, j} \text { and } z_{t}^{\prime}=\sum_{j \in[k]} x_{j} h_{t, j},
$$

where $g_{t, j}$ and $h_{t, j}$ are homogeneous degree-one polynomials in the variables $Y$. Let $f_{t}$ be the non-commutative polynomial

$$
\begin{aligned}
f_{t}=\sum_{m}\left(x_{m} g_{t, m}\right) \sum_{j}\left(x_{j} h_{t, j}\right) & +\sum_{m}\left(x_{m} h_{t, m}\right) \sum_{j}\left(x_{j} g_{t, j}\right) \\
& +\sum_{m}\left(x_{m} \sum_{j}\left(g_{t, j} x_{j}\right) h_{t, m}\right)+\sum_{m}\left(x_{m} \sum_{j}\left(h_{t, j} x_{j}\right) g_{t, m}\right)
\end{aligned}
$$

with summations ranging over $[k]$. The polynomial $f$ is $(X, Y)$-symmetric. We can see that $f_{t}$ is a sum of four central polynomials. It is therefore sufficient to show that

$$
f=\frac{1}{4}\left(f_{1}+\cdots+f_{n}\right) .
$$

First, note that $f_{t}^{(c)}=4 z_{t} z_{t}^{\prime}$ and hence

$$
f^{(c)}=\frac{1}{4}\left(f_{1}^{(c)}+\cdots+f_{n}^{(c)}\right) .
$$

Second, note that if $g$ is $(X, Y)$-symmetric and $\alpha=x_{i_{1}} y_{j_{1}} x_{i_{2}} y_{j_{2}}$ is a non-commutative monomial, then

$$
\operatorname{COEF}_{\alpha^{(c)}}\left(g^{(c)}\right)=N\left(i_{1}, j_{1}, i_{2}, j_{2}\right) \operatorname{COEF}_{\alpha}(g),
$$

where

$$
N\left(i_{1}, j_{1}, i_{2}, j_{2}\right)= \begin{cases}1 & \text { if } i_{1}=i_{2} \text { and } j_{1}=j_{2}, \\ 2 & \text { if } i_{1}=i_{2} \text { and } j_{1} \neq j_{2}, \\ 2 & \text { if } i_{1} \neq i_{2} \text { and } j_{1}=j_{2}, \\ 4 & \text { if } i_{1} \neq i_{2} \text { and } j_{1} \neq j_{2} .\end{cases}
$$

Fix a monomial $\alpha=x_{i_{1}} y_{j_{1}} x_{i_{2}} y_{j_{2}}$ and consider the coefficient of $\alpha$ in the two sides of (4.3). Since $f$ is $(X, Y)$-symmetric, we have

$$
\operatorname{COEF}_{\alpha^{(c)}}\left(f^{(c)}\right)=N\left(i_{1}, j_{1}, i_{2}, j_{2}\right) \operatorname{COEF}_{\alpha}(f) .
$$


Hence (4.4) tells us that

$$
\operatorname{COEF}_{\alpha}(f)=\frac{\operatorname{COEF}_{\alpha^{(c)}}\left(f_{1}^{(c)}+\cdots+f_{n}^{(c)}\right)}{4 N\left(i_{1}, j_{1}, i_{2}, j_{2}\right)} .
$$

Since $f_{t}$ is $(X, Y)$-symmetric, we have $\operatorname{COEF}_{\alpha^{(c)}} f_{t}^{(c)}=N\left(i_{1}, j_{1}, i_{2}, j_{2}\right) \operatorname{COEF}_{\alpha}\left(f_{t}\right)$. Hence

$$
\operatorname{COEF}_{\alpha}(f)=\frac{1}{4}\left(\operatorname{COEF}_{\alpha}\left(f_{1}\right)+\cdots+\operatorname{COEF}_{\alpha}\left(f_{t}\right)\right)
$$

and equation (4.3) follows.

Proof of Theorem 1.7. Recall the definition of the identity polynomial,

$$
\mathrm{ID}_{k}=\sum_{i, j \in[k]} x_{i} y_{j} x_{i} y_{j}
$$

The commutative polynomial $\mathrm{ID}_{k}^{(c)}$ is the polynomial $\mathrm{SOS}_{k}$,

$$
\mathrm{SOS}_{k}=\sum_{i \in[k]} x_{i}^{2} \sum_{j \in[k]} y_{j}^{2} \text {. }
$$

The theorem follows from Propositions 3.2 and 4.1 .

Let us note that it is not necessary to separate variables in $\mathrm{ID}_{k}$ into two disjoint sets $X$ and $Y$. In the non-commutative setting, this is just a cosmetic detail. This is a consequence of a more general phenomenon discussed in Section 7.1.

Remark 4.2. $w\left(\operatorname{ID}_{k}\right)=w\left(\sum_{i, j \in[k]} x_{i} x_{j} x_{i} x_{j}\right)$.

Proof. Denote $g=\sum_{i, j \in[k]} x_{i} x_{j} x_{i} x_{j}$. Clearly $w(g) \leq w\left(\mathrm{ID}_{k}\right)$ and we must prove the opposite inequality. Let $X:=\left\{x_{i}: i \in[k]\right\}$ and write $\operatorname{ID}_{k}$ as $\sum_{i, j \in[k]} x_{i, 0} x_{j, 1} x_{i, 0} x_{j, 1}$ in variables $X_{b}=\left\{x_{i, b}: i \in[k]\right\}, b \in\{0,1\}$. If $f$ is a homogeneous polynomial of degree $r$ in $X$ and $e=\left\langle e_{1}, \ldots, e_{r}\right\rangle \in\{0,1\}^{n}$, let $f^{e}$ be the polynomial s.t. $f^{e}=f^{e}\left[X_{e_{1}}, \ldots, X_{e_{r}}\right]$ and

$$
\operatorname{COEF}_{x_{i_{1}, e_{1}} \ldots x_{i_{r}, e_{r}}}\left(f^{e}\right)=\operatorname{COEF}_{x_{i_{1}} \ldots x_{i_{r}}}(f) .
$$

Hence $\mathrm{ID}_{k}=g^{0101}$. Moreover, if $g=f_{1}+\cdots+f_{n}$, then $g^{0101}=f_{1}^{0101}+\cdots+f_{n}^{0101}$. It is thus sufficient to prove that if $f_{j}$ is central, then $f_{j}^{(0101)}$ is also central. This follows from the following: $(g h)^{(0101)}=g^{(01)} h^{(01)}$ if $g, h$ are homogeneous polynomials of degree two, and $(h g \bar{h})^{(0101)}=h^{(0)} g^{(10)} \bar{h}^{(1)}$ if $h, g, \bar{h}$ are homogeneous polynomials of degrees one, two and one.

\section{Higher Degrees}

In this section, we show that a sufficiently strong lower bound on the width of a degree-four polynomial implies an exponential lower bound on the width, and hence on the circuit-size, of a related high-degree polynomial.

Let $f$ be a homogeneous polynomial of degree $4 r$. We assume that $f$ contains only two variables $z_{0}$ and $z_{1}$. We define $f^{(\lambda)}$ to be the polynomial obtained by replacing degree- $r$ monomials in $f$ by new variables. Formally, for every monomial $\alpha$ of degree $r$ in variables $z_{0}, z_{1}$, introduce a new variable $x_{\alpha}$. The polynomial $f^{(\lambda)}$ is defined as the homogenous degree-four polynomial in the $2^{r}$ variables $X=\left\{x_{\alpha}: \operatorname{deg} \alpha=r\right\}$ 
satisfying

$$
\operatorname{COEF}_{x_{\alpha_{1}} x_{\alpha_{2}} x_{\alpha_{3}} x_{\alpha_{4}}}\left(f^{(\lambda)}\right)=\operatorname{COEF}_{\alpha_{1} \alpha_{2} \alpha_{3} \alpha_{4}}(f) .
$$

Remark 5.1. Let $g$ be a homogeneous degree-four polynomial in $k$ variables. If $k \leq 2^{r}$, then there exists a polynomial $f$ of degree $4 r$ in variables $z_{0}, z_{1}$ such that $g=f^{(\lambda)}$ (up to a renaming of variables).

Proof. For $e=\left(e_{1}, \ldots, e_{r}\right) \in\{0,1\}^{r}$, let $z_{e}$ be the monomial $\prod_{j=1}^{r} z_{e_{j}}$. If $k \leq 2^{r}$ and $i \in[k]$, let $(i) \in\{0,1\}^{r}$ be the binary representation of $i$. If

$$
g=\sum_{i_{1} j_{1} i_{2} j_{2} \in[k]} a_{i_{1} j_{1} i_{2} j_{2}} x_{i_{1}} x_{j_{1}} x_{i_{2}} x_{j_{2}},
$$

let

$$
f=\sum_{i_{1} j_{1} i_{2} j_{2} \in[k]} a_{i_{1} j_{1} i_{2} j_{2}} z_{\left(i_{1}\right)} z_{\left(j_{1}\right)} z_{\left(i_{2}\right)} z_{\left(j_{2}\right)} .
$$

We now relate $w(f)$ and $w\left(f^{(\lambda)}\right)$. To do so, we need a modified version of Proposition 3.2, Let $f$ be a homogeneous polynomial of degree $4 r$. We say that $f$ is block-central, if either

I. $f=g h$, where $g, h$ are homogeneous polynomials with $\operatorname{deg} g=\operatorname{deg} h=2 r$, or

II. $f=\sum_{i \in[m]} h_{i} g \bar{h}_{i}$, where $g, h_{i}, \bar{h}_{i}$ are homogeneous polynomials of degrees $\operatorname{deg} g=2 r$ and $\operatorname{deg} h_{i}=\operatorname{deg} \bar{h}_{i}=r$ for every $i \in[m]$.

Every block-central polynomial is also central. The following lemma shows that every central polynomial can be written as a sum of $2^{r}$ block-central polynomials. The lemma thus enables us to consider a simpler problem, i.e., lower bounding the width with respect to block-central polynomials. However, this simplification comes with a price, namely, a loss of a factor of $2^{r}$.

Lemma 5.2. Let $f$ be a central polynomial of degree $4 r$ in two variables $z_{0}, z_{1}$. Then there exist $n \leq 2^{r}$ and block-central polynomials $f_{1}, \ldots, f_{n}$ so that $f=f_{1}+$ $\cdots+f_{n}$.

Proof. Let $M(k)$ be the set of monomials in variables $z_{0}, z_{1}$ of degree exactly $k$. The size of $M(k)$ is $2^{k}$. As $f$ is central, by Remark 3.1, we can write $f$ as

$$
f=\sum_{\alpha \in M\left(d_{1}\right), \omega \in M\left(d_{2}\right)} c(\alpha, \omega) \alpha G \omega
$$

where $c(\alpha, \omega)$ is a field element, $G$ is a homogeneous polynomial of degree $d_{0}$ with $4 r / 3 \leq d_{0}<8 r / 3$, and $d_{0}+d_{1}+d_{2}=4 r$.

Our goal is to write $f$ as a sum of block-central polynomials; namely, we wish to write $f$ as a sum of polynomials of either type I or type II. We use the parameters $d_{0}, d_{1}, d_{2}$ to determine the type of these polynomials, according to the following case distinction. 
Assume first that $d_{0}+2 d_{1} \leq 3 r$. We express $f$ as a sum of type I polynomials. There are two subcases to consider.

1. $d_{0}+d_{1} \leq 2 r$ : Every monomial $\omega \in M\left(d_{2}\right)$ can be written as $\omega_{1} \omega_{2}$, where $\omega_{1} \in M(t), \omega_{2} \in M\left(d_{2}-t\right)$ and $t=2 r-\left(d_{0}+d_{1}\right)$. Then (5.2) can be written as

$$
f=\sum_{\alpha \in M\left(d_{1}\right), \omega_{1} \in M(t)} f_{\alpha, \omega_{1}},
$$

where

$$
f_{\alpha, \omega_{1}}=\left(\alpha G \omega_{1}\right)\left(\sum_{\omega_{2} \in M\left(d_{2}-t\right)} c\left(\alpha, \omega_{1} \omega_{2}\right) \omega_{2}\right) .
$$

As $d_{2}-t=2 r$, each $f_{\alpha, \omega_{1}}$ is of type I. There are at most $\left|M\left(d_{1}\right)\right||M(t)|=$ $2^{2 r-d_{0}}$ such $f_{\alpha, \omega_{1}}$. Since $d_{0} \geq 4 r / 3$, there are at most $2^{2 r / 3}$ of them.

2. $d_{0}+d_{1}>2 r$ : We can write $G=\sum_{\gamma \in M(t)} G_{\gamma} \gamma$, where $t=d_{0}+d_{1}-2 r$, and $G_{\gamma}$ are some polynomials of degree $d_{0}-t$. Then

$$
f=\sum_{\alpha \in M\left(d_{1}\right), \gamma \in M(t)} f_{\alpha, \gamma}
$$

where

$$
f_{\alpha, \gamma}=\left(\alpha G_{\gamma}\right)\left(\sum_{\omega \in M\left(d_{2}\right)} c(\alpha, \omega) \gamma \omega\right) .
$$

Each $f_{\alpha, \gamma}$ is of type I, and the number of such $f_{\alpha, \gamma}$ is $2^{d_{0}+2 d_{1}-2 r} \leq 2^{r}$, as $d_{0}+2 d_{1} \leq 3 r$.

If $d_{0}+2 d_{2} \leq 3 r$, the argument is analogous. Hence we are in the situation $d_{0}+2 d_{1}>3 r$ and $d_{0}+2 d_{2}>3 r$. In this case, we express $f$ as a sum of central polynomials of type II. There are four subcases to consider.

1. $d_{1} \geq r$ and $d_{2} \geq r$ : For $\alpha \in M\left(d_{1}\right)$, write $\alpha=\alpha_{1} \alpha_{2}$ with $\alpha_{1} \in M(r)$ and $\alpha_{2} \in M\left(d_{1}-r\right)$. For $\omega \in M\left(d_{2}\right)$, write $\omega=\omega_{1} \omega_{2}$ with $\omega_{1} \in M\left(d_{2}-r\right)$ and $\omega_{2} \in M(r)$. Then

$$
f=\sum_{\alpha_{2} \in M\left(d_{1}-r\right), \omega_{1} \in M\left(d_{2}-r\right)} f_{\alpha_{2}, \omega_{1}},
$$

where

$$
f_{\alpha_{2}, \omega_{1}}=\sum_{\alpha_{1}, \omega_{2} \in M(r)} c\left(\alpha_{1} \alpha_{2}, \omega_{1} \omega_{2}\right) \alpha_{1}\left(\alpha_{2} G \omega_{1}\right) \omega_{2} .
$$

Each $f_{\alpha_{2}, \omega_{1}}$ is of type II. There are $2^{2 r-d_{0}} \leq 2^{2 r / 3}$ such $f_{\alpha_{2}, \omega_{1}}$, since $d_{0} \geq$ $4 r / 3$.

2. $d_{1}<r$ and $d_{2} \geq r$ : Write $G=\sum_{\gamma \in M\left(r-d_{1}\right)} \gamma G_{\gamma}$, where $G_{\gamma}$ is a homogeneous polynomial of degree $d_{0}-\left(r-d_{1}\right)$. Write $\omega=\omega_{1} \omega_{2}$ with $\omega_{1} \in$ $M\left(d_{2}-r\right)$ and $\omega_{2} \in M(r)$. Then

$$
f=\sum_{\gamma \in M\left(r-d_{1}\right), \omega_{1} \in M\left(d_{2}-r\right)} f_{\gamma, \omega_{1}},
$$

where

$$
f_{\gamma, \omega_{1}}=\sum_{\alpha \in M\left(d_{1}\right), \omega_{2} \in M(r)} c\left(\alpha, \omega_{1} \omega_{2}\right) \alpha \gamma\left(G_{\gamma} \omega_{1}\right) \omega_{2}
$$


Each $f_{\gamma, \omega_{1}}$ is of type II, and there are $2^{d_{2}-d_{1}}<2^{r}$ such $f_{\gamma, \omega_{1}}$, since $d_{0}+$ $2 d_{1}>3 r$.

3. $d_{1} \geq r$ and $d_{2}<r$ : This is the previous case with $d_{2}$ and $d_{1}$ interchanged.

4. $d_{1}<r$ and $d_{2}<r$ : Write $G=\sum_{\gamma_{1} \in M\left(r-d_{1}\right), \gamma_{2} \in M\left(r-d_{2}\right)} \gamma_{1} G_{\gamma_{1}, \gamma_{2}} \gamma_{2}$, where $G_{\gamma_{1}, \gamma_{2}}$ is a homogeneous polynomial of degree $2 r$. Then

$$
f=\sum_{\gamma_{1} \in M\left(r-d_{1}\right), \gamma_{2} \in M\left(r-d_{2}\right)} f_{\gamma_{1}, \gamma_{2}}
$$

where

$$
f_{\gamma_{1}, \gamma_{2}}=\sum_{\alpha \in M\left(d_{1}\right), \omega \in M\left(d_{2}\right)} c(\alpha, \omega) \alpha \gamma_{1} G_{\gamma_{1}, \gamma_{2}} \gamma_{2} \omega .
$$

Each $f_{\gamma_{1}, \gamma_{2}}$ is of type II, and there are $2^{d_{0}-2 r} \leq 2^{2 r / 3}$ such $f_{\gamma_{1}, \gamma_{2}}$, since $d_{0} \leq 8 r / 3$.

We can now relate the width of $f$ and $f^{(\lambda)}$.

Proposition 5.3. Let $f$ be a homogeneous polynomial of degree $4 r$ in the variables $z_{0}, z_{1}$. Then $w(f) \geq 2^{-r} w\left(f^{(\lambda)}\right)$.

Proof. Assume $w(f)=n$. Lemma 5.2 implies that $f=f_{1}+\cdots+f_{n^{\prime}}$, where $n^{\prime} \leq 2^{r} n$ and $f_{j}$ are block-central polynomials. Equation (5.1) implies that

$$
f^{(\lambda)}=f_{1}^{(\lambda)}+\cdots+f_{n^{\prime}}^{(\lambda)} .
$$

It is thus sufficient to show that every $f_{t}^{(\lambda)}$ is a central polynomial, for then $w\left(f^{(\lambda)}\right) \leq n^{\prime} \leq 2^{r} n$.

In order to do so, let us extend the definition of $(.)^{(\lambda)}$ as follows. If $g$ is a polynomial of degree $\ell r$ in the variables $z_{0}, z_{1}$, let $g^{(\lambda)}$ be the homogeneous polynomial of degree $\ell$ in $X$ so that

$$
\operatorname{coEF}_{x_{\alpha_{1}} \cdots x_{\alpha_{k}}}\left(g^{(\lambda)}\right)=\operatorname{COEF}_{\alpha_{1} \cdots \alpha_{k}}(g) .
$$

If $g, h$ are homogeneous polynomials whose degree is divisible by $r$, we obtain $(g h)^{(\lambda)}=g^{(\lambda)} h^{(\lambda)}$. Hence if $f_{t}=g_{t} h_{t}$ a block-central polynomial of type I, then $f_{t}^{(\lambda)}=g_{t}^{(\lambda)} h_{t}^{(\lambda)}$ is a central polynomial of type (a) according to Lemma 3.5 with $X=X_{1}=X_{2}=X_{3}=X_{4}$. If $f_{t}=\sum_{i} h_{t, i} g_{t} \bar{h}_{t, i}$ is a block-central polynomial of type II, $f_{t}^{(\lambda)}=\sum_{i} h_{t, i}^{(\lambda)} g_{t}^{(\lambda)} \bar{h}_{t, i}(\lambda)$, and hence $f_{t}^{(\lambda)}$ is a central polynomial of type (b) according to Lemma 3.5

By Remark 5.1, we can start with a degree-four polynomial in $k \leq 2^{r}$ variables and "lift" it to a polynomial $f$ of degree $4 r$ such that $f^{(\lambda)}=g$. We can then deduce that a sufficiently strong lower bound on the bilinear complexity of $g$ implies an exponential lower bound for the circuit complexity of $f$. We apply this to the specific case of the identity polynomial. The lifted identity polynomial, $\mathrm{LID}_{r}$, is the polynomial in variables $z_{0}, z_{1}$ of degree $4 r$ defined by

$$
\mathrm{LID}_{r}=\sum_{e \in\{0,1\}^{2 r}} z_{e} z_{e}
$$

where for $e=\left(e_{1}, \ldots, e_{s}\right) \in\{0,1\}^{s}$, we define $z_{e}=\prod_{i=1}^{s} z_{e_{i}}$. 
Corollary 5.4 (Corollary 2.3 restated). If $\mathcal{B}\left(\mathrm{SOS}_{k}\right) \geq \Omega\left(k^{1+\epsilon}\right)$ for some $\epsilon>0$, then $\mathcal{C}\left(\mathrm{LID}_{r}\right) \geq 2^{\Omega(r)}$. In general, $\mathcal{C}\left(\mathrm{LID}_{r}\right) \geq \Omega\left(\frac{\mathcal{B}\left(\mathrm{SOS}_{2 r}\right)}{r^{3} 2^{r}}\right)$.

Proof. The definition of $\mathrm{LID}_{r}$ can be equivalently written as

$$
\operatorname{LID}_{r}=\sum_{e_{1}, e_{2} \in\{0,1\}^{r}} z_{e_{1}} z_{e_{2}} z_{e_{1}} z_{e_{2}} .
$$

By definition, $\operatorname{LID}_{r}^{(\lambda)}=\sum_{i, j \in[k]} x_{i} x_{j} x_{i} x_{j}$ with $k=2^{r}$. Hence, by Remark 4.2, $w\left(\operatorname{LID}_{r}^{(\lambda)}\right)=w\left(\operatorname{ID}_{k}\right)$. By Proposition 5.3, $w\left(\operatorname{LID}_{r}\right) \geq 2^{-r} w\left(\operatorname{LID}_{r}^{(\lambda)}\right)$. Hence $w\left(\operatorname{LID}_{r}\right)$ $\geq 2^{-r} w\left(\mathrm{ID}_{k}\right)$. By definition, $\mathrm{SOS}_{k}=\mathrm{ID}_{k}^{(c)}$. Hence, by Proposition 4.1 $w\left(\mathrm{ID}_{k}\right) \geq$ $\mathcal{B}\left(\mathrm{SOS}_{k}\right)$. Hence $w\left(\mathrm{LID}_{r}\right) \geq 2^{-r} \mathcal{B}\left(\mathrm{SOS}_{k}\right)$ with $k=2^{r}$. By Proposition 3.2 $\mathcal{C}\left(\mathrm{LID}_{r}\right) \geq \Omega\left(r^{-3} w\left(\mathrm{LID}_{r}\right)\right) \geq \Omega\left(r^{-3} 2^{-r} \mathcal{B}\left(\mathrm{SOS}_{2^{r}}\right)\right)$.

Finally, if $\mathcal{B}\left(\mathrm{SOS}_{k}\right) \geq \Omega\left(k^{1+\epsilon}\right)$ for some $\epsilon>0$, then we have $\mathcal{C}\left(\mathrm{LID}_{r}\right) \geq$ $\Omega\left(r^{-3} 2^{-r} 2^{r(1+\epsilon)}\right)=\Omega\left(r^{-3} 2^{\epsilon r}\right)$, and so $\mathcal{C}\left(\operatorname{LID}_{r}\right) \geq 2^{\Omega(r)}$.

One motivation for studying the lifted identity polynomial is that we believe it is hard for non-commutative circuits. However, note that an apparently similar polynomial has small circuit-size. For $e=\left(e_{1}, \ldots, e_{s}\right) \in\{0,1\}^{s}$, let $e^{\star}=\left(e_{s}, \ldots, e_{1}\right)$. The polynomial

$$
\sum_{e \in\{0,1\}^{2 r}} z_{e} z_{e^{\star}}
$$

has a non-commutative circuit of linear size. This result can be found in [23], where it is also shown that the non-commutative formula complexity of this polynomial is exponential in $r$.

We now show that $\operatorname{LID}_{r}$ is reducible to the permanent of dimension $4 r$.

Lemma 5.5 (Lemma 2.4 restated). There exists a matrix $M$ of dimension $4 r \times 4 r$ whose non-zero entries are variables $z_{0}, z_{1}$ so that the permanent of $M$ is $\operatorname{LID}_{r}$.

Proof. For $j \in\{0,1\}$, let $D_{j}$ be the $2 r \times 2 r$ matrix with $z_{j}$ on the diagonal and zero everywhere else. The matrix $M$ is defined as

$$
M=\left[\begin{array}{cc}
D_{0} & D_{1} \\
D_{1} & D_{0}
\end{array}\right]
$$

The permanent of $M$ taken row by row is

$$
\operatorname{PERM}(M)=\sum_{\sigma} M_{1, \sigma(1)} M_{2, \sigma(2)} \cdots M_{4 r, \sigma(4 r)},
$$

where $\sigma$ is a permutation of $[4 r]$. The permutations that give non-zero value in $\operatorname{PERM}(M)$ satisfy: for every $i \in[2 r]$, if $\sigma(i)=i$, then $\sigma(2 r+i)=2 r+i$, and if $\sigma(i)=2 r+i$, then $\sigma(2 r+i)=i$. By definition of $M$, this means that for every such $\sigma$ and $i \in[2 r], M_{i, \sigma(i)}=M_{i+2 r, \sigma(i+2 r)}$. Moreover, given the values of such a $\sigma$ on $[2 r]$, it can be uniquely extended to all of $[4 r]$.

Theorem 1.8 follows from Corollary 2.3 and Lemma 2.4 
5.1. Explicit polynomials and completeness of the non-commutative permanent. The (conditional) exponential lower bound on the circuit-size of the permanent can be significantly generalized. An important property that the noncommutative permanent shares with its commutative counterpart is completeness for the class of explicit polynomials. This enables us to argue that a superlinear lower bound on the width of an explicit degree-four polynomial implies an exponential lower bound on the permanent.

Let $\left\{f_{k}\right\}$ be an infinite family of non-commutative polynomials over $\mathbb{F}$ so that every $f_{k}$ has at most $p(k)$ variables and degree at most $p(k)$, where $p: \mathbb{N} \rightarrow \mathbb{N}$ is a polynomial. We call $\left\{f_{k}\right\}$ explicit if there exists a polynomial-time algorithm which, given $k$ and a monomial $\alpha$ as input, computes $\operatorname{COEF}_{\alpha}\left(f_{k}\right)$. Hence $\mathrm{PERM}_{k}$ and other families of polynomials are explicit in this sense. In the commutative setting, the following theorem is a consequence of the VNP completeness of the permanent, as given in [35. In the non-commutative setting, one can prove a similar result [12].

Theorem 5.6. Assume that $\left\{f_{k}\right\}$ is an explicit family of non-commutative polynomials such that $\mathcal{C}\left(f_{k}\right) \geq 2^{\Omega(k)}$. Then $\mathcal{C}\left(\mathrm{PERM}_{k}\right) \geq 2^{\Omega(k)}$.

Proof of Theorem 1.9. For a commutative biquadratic polynomial in $k$ variables,

$$
f=\sum_{i_{1} \leq i_{2} \in[k], j_{1} \leq j_{2} \in[k]} a_{i_{1}, j_{1}, i_{2}, j_{2}} x_{i_{1}} y_{j_{1}} x_{i_{2}} y_{j_{2}},
$$

define $f^{\prime}$ as the non-commutative polynomial

$$
f^{\prime}=\sum_{i_{1}, j_{1}, i_{2}, j_{2} \in[k]} a_{i_{1}, j_{1}, i_{2}, j_{2}} x_{i_{1}} y_{j_{1}} x_{i_{2}} y_{j_{2}} .
$$

This is to guarantee that $f^{\prime}=f^{\prime}[X, Y, X, Y]$ and $\left(f^{\prime}\right)^{(c)}=f$ is as required in Proposition 4.1. Let $r$ be the smallest integer so that $2^{r} \geq 2 k$. Let $f^{\star}$ be the polynomial given by Remark 5.1 so that $\left(f^{\star}\right)^{(\lambda)}=f^{\prime}$. If $f$ is explicit, $f^{\star}$ is explicit.

Let $\left\{f_{k}\right\}$ be as in the assumption. As in the proof of Corollary 2.3. we conclude that $f_{k}^{\star}$ require exponential-size non-commutative circuits. By Theorem 5.6, this implies an exponential lower bound for the permanent.

\section{REAL SUM-OF-SQUARES}

In this section, we prove Theorem 1.10, We construct a real biquadratic polynomial $f$ in the variables $X=\left\{x_{1}, \ldots, x_{k}\right\}$ and $Y=\left\{y_{1}, \ldots, y_{k}\right\}$ over $\mathbb{R}$, so that $f$ can be written as $f=\sum_{i \in[n]} z_{i}^{2}$ with $z_{i}$ bilinear in $X, Y$, but every such $n$ is at least $k^{2} / 16$. The construction of $f$ is in polynomial time with respect to the length of the binary representation of $k$.

Remark 6.1. In the case of $\mathbb{R}$, the condition that the $z_{i}$ are bilinear is satisfied automatically, provided $z_{i}$ is a polynomial.

Recall that in this section, all polynomials are commutative.

6.1. Real sum-of-squares and vector matrices. We phrase the problem of lower bounding $\mathcal{S}_{\mathbb{R}}(f)$ in terms of matrices of real vectors. Let $V=\left\{\mathbf{v}_{i, j}: i \in\right.$ $[r], j \in[s]\}$ be a matrix whose entries are vectors in $\mathbb{R}^{n}$. We call $V$ a vector matrix, and $n$ is called the height of $V$. Let $U=\left\{\mathbf{u}_{i, j}: i \in[r], j \in[s]\right\}$ be a vector matrix 
of arbitrary height. We say that $U$ and $V$ are equivalent if for every $i_{1}, i_{2} \in[r]$, $j_{1}, j_{2} \in[s]$,

$$
\mathbf{v}_{i_{1}, j_{1}} \cdot \mathbf{v}_{i_{2}, j_{2}}=\mathbf{u}_{i_{1}, j_{1}} \cdot \mathbf{u}_{i_{2}, j_{2}}
$$

where for two vectors $\mathbf{w}_{1}, \mathbf{w}_{2}$ in $\mathbb{R}^{m}, \mathbf{w}_{1} \cdot \mathbf{w}_{2}$ is the standard inner product in $\mathbb{R}^{m}$. We say that $U$ and $V$ are similar if for every $i_{1}, i_{2} \in[r]$ and $j_{1}, j_{2} \in[s]$,

$$
\mathbf{v}_{i_{1}, j_{1}} \cdot \mathbf{v}_{i_{2}, j_{2}}+\mathbf{v}_{i_{1}, j_{2}} \cdot \mathbf{v}_{i_{2}, j_{1}}=\mathbf{u}_{i_{1}, j_{1}} \cdot \mathbf{u}_{i_{2}, j_{2}}+\mathbf{u}_{i_{1}, j_{2}} \cdot \mathbf{u}_{i_{2}, j_{1}} \cdot
$$

It is more convenient to consider the four different cases of this equality:

$$
\begin{aligned}
& \mathbf{v}_{i, j} \cdot \mathbf{v}_{i, j}=\mathbf{u}_{i, j} \cdot \mathbf{u}_{i, j} . \\
& \mathbf{v}_{i, j_{1}} \cdot \mathbf{v}_{i, j_{2}}=\mathbf{u}_{i, j_{1}} \cdot \mathbf{u}_{i, j_{2}} \text { if } j_{1} \neq j_{2} . \\
& \mathbf{v}_{i_{1}, j} \cdot \mathbf{v}_{i_{2}, j}=\mathbf{u}_{i_{1}, j} \cdot \mathbf{u}_{i_{1}, j} \text { if } i_{1} \neq i_{2} . \\
& \mathbf{v}_{i_{1}, j_{1}} \cdot \mathbf{v}_{i_{2}, j_{2}}+\mathbf{v}_{i_{1}, j_{2}} \cdot \mathbf{v}_{i_{2}, j_{1}}=\mathbf{u}_{i_{1}, j_{1}} \cdot \mathbf{u}_{i_{2}, j_{2}}+\mathbf{u}_{i_{1}, j_{2}} \cdot \mathbf{u}_{i_{2}, j_{1}} \text { if } i_{1} \neq i_{2}, j_{1} \neq j_{2} .
\end{aligned}
$$

A $k \times k$ vector matrix $V$ defines a polynomial $f(V)$ in the variables $X, Y$ by

$$
f(V)=\sum_{i_{1} \leq i_{2}, j_{1} \leq j_{2}} a_{i_{1}, i_{2}, j_{1}, j_{2}} x_{i_{1}} x_{i_{2}} y_{j_{1}} y_{j_{2}},
$$

with

$$
a_{i_{1}, i_{2}, j_{1}, j_{2}}=\left\{\begin{array}{cl}
\mathbf{v}_{i, j} \cdot \mathbf{v}_{i, j} & \text { if } i_{1}=i_{2}=i, j_{1}=i_{2}=j, \\
2 \mathbf{v}_{i, j_{1}} \cdot \mathbf{v}_{i, j_{2}} & \text { if } i_{1}=i_{2}=i, j_{1}<j_{2}, \\
2 \mathbf{v}_{i_{1}, j} \cdot \mathbf{v}_{i_{2}, j} & \text { if } i_{1}<i_{2}, j_{1}=j_{2}=j, \\
2\left(\mathbf{v}_{i_{1}, j_{1}} \cdot \mathbf{v}_{i_{2}, j_{2}}+\mathbf{v}_{i_{1}, j_{2}} \cdot \mathbf{v}_{i_{2}, j_{1}}\right) & \text { if } i_{1}<i_{2}, j_{1}<j_{2} .
\end{array}\right.
$$

Note that if $U$ and $V$ are similar, then $f(U)=f(V)$.

Lemma 6.2. Let $V$ be $a k \times k$ vector matrix. Then the following are equivalent:

(1) There exist bilinear forms $z_{1}, \ldots, z_{n}$ so that $f(V)=\sum_{i \in[n]} z_{i}^{2}$.

(2) There exists a vector matrix $U$ of height $n$ so that $U$ and $V$ are similar.

The lemma shows that $f(V)$ can always be written as a sum of real bilinear squares. Moreover, the proof of the lemma entails the converse: if $f$ can be written as a sum of bilinear squares, then $f=f(V)$ for some vector matrix $V$.

Proof. Assume that

$$
f(V)=\sum_{i \in[n]} z_{i}^{2}
$$

where each $z_{i}$ is bilinear. For $\ell \in[n]$ and $i, j \in[k]$, let $u_{i, j}[\ell]$ be the coefficient of $x_{i} y_{j}$ in $z_{\ell}$, and let $\mathbf{u}_{i, j}=\left(u_{i, j}[1], \ldots, u_{i, j}[n]\right)$. Let $U=\left\{\mathbf{u}_{i, j}: i, j \in[k]\right\}$ be the $k \times k$ vector matrix of height $n$. Equation (6.6) can be written as

$$
f(V)=\left(\sum_{i, j \in[k]} \mathbf{u}_{i, j} x_{i} y_{j}\right) \cdot\left(\sum_{i, j \in[k]} \mathbf{u}_{i, j} x_{i} y_{j}\right) .
$$


The right-hand side of (6.7) can be written as

$$
\begin{aligned}
& \sum_{i, j}\left(\left(\mathbf{u}_{i, j} \cdot \mathbf{u}_{i, j}\right) x_{i}^{2} y_{j}^{2}\right)+2 \sum_{i, j_{1}<j_{2}}\left(\left(\mathbf{u}_{i, j_{1}} \cdot \mathbf{u}_{i, j_{2}}\right) x_{i}^{2} y_{j_{1}} y_{j_{2}}\right) \\
& +2 \sum_{i_{1}<i_{2}, j}\left(\left(\mathbf{u}_{i_{1}, j} \cdot \mathbf{u}_{i_{2}, j}\right) x_{i_{1}} x_{i_{2}} y_{j}^{2}\right) \\
& \quad+2 \sum_{i_{1}<i_{2}, j_{1}<j_{2}}\left(\left(\mathbf{u}_{i_{1}, j_{1}} \cdot \mathbf{u}_{i_{2}, j_{2}}+\mathbf{u}_{i_{1}, j_{2}} \cdot \mathbf{u}_{i_{2}, j_{1}}\right) x_{i_{1}} x_{i_{2}} y_{j_{1}} y_{j_{2}}\right) .
\end{aligned}
$$

Comparing the coefficients on the left-hand and right-hand sides of (․․7), we obtain

$$
a_{i_{1}, i_{2}, j_{1}, j_{2}}=\left\{\begin{array}{cl}
\mathbf{u}_{i, j} \cdot \mathbf{u}_{i, j} & \text { if } i_{1}=i_{2}=i, j_{1}=i_{2}=j, \\
2 \mathbf{u}_{i, j_{1}} \cdot \mathbf{u}_{i, j_{2}} & \text { if } i_{1}=i_{2}=i, j_{1}<j_{2}, \\
2 \mathbf{u}_{i_{1}, j} \cdot \mathbf{u}_{i_{2}, j} & \text { if } i_{1}<i_{2}, j_{1}=j_{2}=j, \\
2\left(\mathbf{u}_{i_{1}, j_{1}} \cdot \mathbf{u}_{i_{2}, j_{2}}+\mathbf{u}_{i_{1}, j_{2}} \cdot \mathbf{u}_{i_{2}, j_{1}}\right) & \text { if } i_{1}<i_{2}, j_{1}<j_{2} .
\end{array}\right.
$$

By (6.5), this means that $U$ and $V$ are similar. Conversely, if $U$ is a vector matrix similar to $V$, we obtain (6.6) by means of (6.5), (6.7) and (6.8).

6.2. A hard vector matrix. In this section we construct a hard polynomial by describing its vector matrix $M$. Let $\mathbf{e}_{i}(j), i, j \in[\ell]$, be $\ell^{2}$ orthonormal vectors in $\mathbb{R}^{\ell^{2}}$. The matrix $M$ will be a $k \times k$ matrix with $k=4(\ell-1)$ whose entries are vectors $\mathbf{e}_{i}(j)$.

For $t \in[\ell]$, let $L(t)$ be the following $2 \times 2(\ell-1)$ matrix:

$$
\begin{aligned}
& L(t)= \\
& {\left[\begin{array}{cccccccccc}
\mathbf{e}_{1}(t) & \mathbf{e}_{1}(t) & \mathbf{e}_{2}(t) & \mathbf{e}_{2}(t) & \mathbf{e}_{3}(t) & \mathbf{e}_{3}(t) & \cdots & \mathbf{e}_{\ell-2}(t) & \mathbf{e}_{\ell-1}(t) & \mathbf{e}_{\ell-1}(t) \\
\mathbf{e}_{1}(t) & \mathbf{e}_{2}(t) & \mathbf{e}_{2}(t) & \mathbf{e}_{3}(t) & \mathbf{e}_{3}(t) & \mathbf{e}_{4}(t) & \cdots & \mathbf{e}_{\ell-1}(t) & \mathbf{e}_{\ell-1}(t) & \mathbf{e}_{\ell}(t)
\end{array}\right] .}
\end{aligned}
$$

Let $M$ be the matrix

$$
M=\left[\begin{array}{cc}
L(1) & L(1) \\
L(1) & L(2) \\
L(2) & L(2) \\
\cdots & \cdots \\
L(\ell-2) & L(\ell-1) \\
L(\ell-1) & L(\ell-1) \\
L(\ell-1) & L(\ell)
\end{array}\right] .
$$

The following theorem shows that $f(M)$ is hard, in the sense of sum-of-squares complexity.

Theorem 6.3. $\mathcal{S}_{\mathbb{R}}(f(M))=\ell^{2}$.

The proof is based on the following lemma.

Lemma 6.4. If $U$ and $M$ are similar, then $U$ and $M$ are equivalent.

Let us first show that Lemma 6.4 implies Theorem 6.3. The matrix $M$ consists of $\ell^{2}$ orthonormal vectors. Hence any matrix $U$ equivalent to $M$ has height at least $\ell^{2}$. The theorem follows from Lemmas 6.4 and 6.2 .

We now proceed to prove Lemma 6.4. Let us state two more definitions. A vector matrix $V$ is called normal if for every $\mathbf{v}$ in $V$, we have $\mathbf{v} \cdot \mathbf{v}=1$. Two vector matrices $V_{1}, V_{2}$ of the same height are called orthogonal if for every $\mathbf{v}_{1}$ in $V_{1}$ and $\mathbf{v}_{2}$ in $V_{2}$, we have $\mathbf{v}_{1} \cdot \mathbf{v}_{2}=0$. 
The proof of Lemma 6.4 starts with the following three simple claims. In the claims, we denote elements of $V, U$ by $\mathbf{v}, \mathbf{u}$, and elements of $V_{p}, U_{p}$ by $\mathbf{v}^{p}, \mathbf{u}^{p}$, where $p$ is an integer. In a crucial way, we employ the following property of real vectors: if $\mathbf{v} \cdot \mathbf{v}=\mathbf{u} \cdot \mathbf{u}=1$ and $\mathbf{v} \cdot \mathbf{u}=1$, then $\mathbf{v}=\mathbf{u}$.

Claim 6.5. If $U$ and $V$ are similar and $V$ is normal, then $U$ is normal.

Proof. This follows from condition (6.1).

Claim 6.6. Let

$$
V=\left[\begin{array}{l}
V_{1} \\
V_{1}
\end{array}\right] \text { and } U=\left[\begin{array}{l}
U_{1} \\
U_{2}
\end{array}\right]
$$

If $V$ is normal and $U$ and $V$ are similar, then $U_{1}=U_{2}$. The same holds for $V=\left[V_{1} V_{1}\right]$ and $U=\left[U_{1} U_{2}\right]$.

Proof. Let $V_{1}$ be an $r \times c$ vector matrix. For every $i \in[r]$ and $j \in[c], \mathbf{v}_{i+r, j}=\mathbf{v}_{i, j}$ and so $\mathbf{v}_{i+r, j} \cdot \mathbf{v}_{i, j}=1$. Since $V$ is normal and $U$ and $V$ are similar, by (6.1), $\mathbf{u}_{i, j} \cdot \mathbf{u}_{i, j}=\mathbf{u}_{i+r, j} \cdot \mathbf{u}_{i+r, j}=1$. By (6.3), $\mathbf{u}_{i+r, j} \cdot \mathbf{u}_{i, j}=1$, and so $\mathbf{u}_{i+r, j}=\mathbf{u}_{i, j}$.

Claim 6.7. Let

$$
V=\left[\begin{array}{ll}
V_{1} & V_{2} \\
V_{3} & V_{4}
\end{array}\right] \text { and } U=\left[\begin{array}{cc}
U_{1} & U_{2} \\
U_{3} & U_{4}
\end{array}\right]
$$

with $V$ a normal matrix. Assume that $V_{1}$ and $V_{4}$ are orthogonal and either

(1) $V_{2}, V_{3}$ are orthogonal and $U_{2}, U_{3}$ are orthogonal, or

(2) $V_{1}=V_{2}=V_{3}, U_{1}=U_{2}=U_{3}$ and $U_{1}, V_{1}$ are equivalent.

Then $U_{1}$ and $U_{4}$ are orthogonal.

Proof. Let $V_{1}$ and $V_{4}$ be of sizes $r_{1} \times c_{1}$ and $r_{2} \times c_{2}$. From condition (6.4), we have that for every $i_{1} \in\left[r_{1}\right], j_{1} \in\left[c_{1}\right], i_{1} \in\left[r_{2}\right]$ and $j_{2} \in\left[c_{2}\right]$,

$$
\mathbf{v}_{i_{1}, j_{1}} \cdot \mathbf{v}_{r_{1}+i_{2}, c_{1}+j_{2}}+\mathbf{v}_{i_{1}, c_{1}+j_{2}} \cdot \mathbf{v}_{r_{1}+i_{2}, j_{1}}=\mathbf{u}_{i_{1}, j_{1}} \cdot \mathbf{u}_{r_{1}+i_{2}, c_{1}+j_{2}}+\mathbf{u}_{i_{1}, c_{1}+j_{2}} \cdot \mathbf{u}_{r_{1}+i_{2}, j_{1}},
$$

which gives

$$
\mathbf{v}_{i_{1}, j_{1}}^{1} \cdot \mathbf{v}_{i_{2}, j_{2}}^{4}+\mathbf{v}_{i_{1}, j_{2}}^{2} \cdot \mathbf{v}_{i_{2}, j_{1}}^{3}=\mathbf{u}_{i_{1}, j_{1}}^{1} \cdot \mathbf{u}_{i_{2}, j_{2}}^{4}+\mathbf{u}_{i_{1}, j_{2}}^{2} \cdot \mathbf{u}_{i_{2}, j_{1}}^{3} \cdot
$$

The property that is common to both cases in the assumption of the claim is that $\mathbf{v}_{i_{1}, j_{2}}^{2} \cdot \mathbf{v}_{i_{2}, j_{1}}^{3}=\mathbf{u}_{i_{1}, j_{2}}^{2} \cdot \mathbf{u}_{i_{2}, j_{1}}^{3}$. Therefore, $\mathbf{u}_{i_{1}, j_{1}}^{1} \cdot \mathbf{u}_{i_{2}, j_{2}}^{4}=\mathbf{v}_{i_{1}, j_{1}}^{1} \cdot \mathbf{v}_{i_{2}, j_{2}}^{4}=0$.

The three claims imply the following, using the special structure of the matrix $L$.

Claim 6.8. If a vector matrix $U$ is similar to $L(t)$, then $U$ is equivalent to $L(t)$.

Proof. Since $L(t)$ is normal, so is $U$ by Claim 6.5. We apply Claim 6.6 to submatrices of $L(t)$ with the form $\left(\mathbf{e}_{i}(t), \mathbf{e}_{i}(t)\right)$ and $\left(\begin{array}{l}\mathbf{e}_{i}(t) \\ \mathbf{e}_{i}(t)\end{array}\right)$, and the corresponding submatrices of $U$, to conclude that $U$ is of the form

$$
U=\left[\begin{array}{lllllllllc}
\mathbf{u}_{1} & \mathbf{u}_{1} & \mathbf{u}_{2} & \mathbf{u}_{2} & \mathbf{u}_{3} & \mathbf{u}_{3} & \cdots & \mathbf{u}_{\ell-2} & \mathbf{u}_{\ell-1} & \mathbf{u}_{\ell-1} \\
\mathbf{u}_{1} & \mathbf{u}_{2} & \mathbf{u}_{2} & \mathbf{u}_{3} & \mathbf{u}_{3} & \mathbf{u}_{4} & \cdots & \mathbf{u}_{\ell-1} & \mathbf{u}_{\ell-1} & \mathbf{u}_{\ell}
\end{array}\right] .
$$

It is thus sufficient to prove that for every $i<j$ in [ $\ell$, the two vectors $\mathbf{u}_{i}$ and $\mathbf{u}_{j}$ are orthogonal. This follows by induction on $j$. If $j=i+1$, apply case (2) of Claim 6.7 to the matrices

$$
\left[\begin{array}{ll}
\mathbf{e}_{i}(t) & \mathbf{e}_{i}(t) \\
\mathbf{e}_{i}(t) & \mathbf{e}_{j}(t)
\end{array}\right] \text { and }\left[\begin{array}{ll}
\mathbf{u}_{i} & \mathbf{u}_{i} \\
\mathbf{u}_{i} & \mathbf{u}_{j}
\end{array}\right]
$$


Otherwise, $j \geq i+2$ and $U$ contains a submatrix

$$
\left[\begin{array}{cc}
\mathbf{u}_{i} & \mathbf{u}_{j-1} \\
\mathbf{u}_{i} & \mathbf{u}_{j}
\end{array}\right]
$$

which is similar to the matrix

$$
\left[\begin{array}{cc}
\mathbf{e}_{i}(t) & \mathbf{e}_{j-1}(t) \\
\mathbf{e}_{i}(t) & \mathbf{e}_{j}(t)
\end{array}\right]
$$

By assumption $\mathbf{u}_{i}$ and $\mathbf{u}_{j-1}$ are orthogonal. Now case (1) of Claim 6.7 implies that $\mathbf{u}_{i}$ and $\mathbf{u}_{j}$ are orthogonal.

Proof of Lemma 6.4. If $U$ is similar to $M$, by Claim 6.8, $U$ has the form

$$
U=\left[\begin{array}{cc}
U(1) & U(1) \\
U(1) & U(2) \\
U(2) & U(2) \\
\cdots & \cdots \\
U(\ell-2) & U(\ell-1) \\
U(\ell-1) & U(\ell-1) \\
U(\ell-1) & U(\ell)
\end{array}\right]
$$

where $U(t)$ is equivalent to $L(t)$. It is now sufficient to prove that $U(i)$ and $U(j)$ are orthogonal whenever $i<j$. This follows by a similar argument to the one in Claim 6.8.

\section{MUltilinear AND ORDERED CIRCUITS}

7.1. Ordered circuits. An interesting property of non-commutative polynomials and circuits is that we can treat occurrences of $x$ at different positions as distinct variables. For example, we could have defined the identity polynomial as a polynomial in $4 k$ variables

$$
\mathrm{ID}_{k}{ }^{\prime}=\sum_{i, j \in[k]} x_{1, i} x_{2, j} x_{3, i} x_{4, j},
$$

or as the polynomial in only $k$ variables

$$
\mathrm{ID}_{k}{ }^{\prime \prime}=\sum_{i, j \in[k]} x_{i} x_{j} x_{i} x_{j} .
$$

These modifications are not important in the non-commutative setting; the circuit complexities of $\mathrm{ID}_{k}, \mathrm{ID}_{k}{ }^{\prime}$ and $\mathrm{ID}_{k}{ }^{\prime \prime}$ differ by at most a constant factor. We discuss this phenomenon in this section.

A homogeneous polynomial $f$ of degree $r$ is called ordered if there exist disjoint sets of variables $X_{1}, \ldots, X_{r}$ so that $f=f\left[X_{1}, \ldots, X_{r}\right]$ with the definition of $f\left[X_{1}, \ldots, X_{r}\right]$ from Section 3.2 In other words, $f$ is ordered if every variable that occurs at position $i$ in some monomial in $f$ is in $X_{i}$ (and the $X_{i}$ 's are disjoint).

An interval $I$ is a set of the form $I=\left[j_{1}, j_{2}\right]=\left\{i: j_{1} \leq i \leq j_{2}\right\}$. A polynomial $g$ is of type $\left[j_{1}, j_{2}\right]$ if $g=g\left[X_{j_{1}}, \ldots, X_{j_{2}}\right]$. It is a homogeneous polynomial of degree $j_{2}-j_{1}+1$. A constant polynomial is of type $I=\emptyset$.

We now define ordered circuits, which are a natural model for computing ordered polynomials. In an ordered circuit $\Phi$, every gate $v$ is associated with an interval $I_{v}=I_{v}(\Phi) \subseteq[r]$. A circuit $\Phi$ is called ordered if it satisfies the following properties:

(1) Every gate $v$ in $\Phi$ computes a polynomial of type $I_{v}$.

(2) If $v=v_{1}+v_{2}$, then $I_{v}=I_{v_{1}}=I_{v_{2}}$. 
(3) If $v=v_{1} \times v_{2}$ with $I_{v}=[i, j]$, then there exists $i-1 \leq \ell \leq j$ so that $I_{v_{1}}=[i, \ell]$ and $I_{v_{2}}=[\ell+1, j]$ (recall that $[i, i-1]=\emptyset$ ).

We can also define an ordered version of a general polynomial. Let $f$ be a homogeneous polynomial of degree $r$ in the variables $X=\left\{x_{1}, \ldots, x_{k}\right\}$. We define the ordered version of $f$, denoted $f^{(\text {ord })}$, as follows. For every $j \in[r]$ and $i \in$ $[k]$, introduce a new variable $x_{j, i}$, and let $X_{j}=\left\{x_{j, 1}, \ldots, x_{j, k}\right\}$. For a monomial $\alpha=x_{i_{1}} \ldots x_{i_{r}}$, let $\alpha^{(\text {ord })}:=x_{1, i_{1}} \ldots x_{r, i_{r}}$. The polynomial $f^{(\text {ord })}$ is the ordered polynomial in the variables $X_{1}, \ldots, X_{r}$ defined by

$$
\operatorname{COEF}_{\alpha(\text { ord })}\left(f^{(\text {ord })}\right)=\operatorname{COEF}_{\alpha}(f) \text {. }
$$

Given $f^{(o r d)}$ we can easily recover $f$ by substituting $x_{j, i}=x_{i}$ for every $j \in[r]$ and $i \in[k]$. When $f$ is already ordered, then $f^{(\text {ord })}$ and $f$ are the same polynomials, up to renaming of variables.

The following theorem shows that non-commutative circuits computing $f$ can be efficiently simulated by ordered circuits computing $f^{(\text {ord })}$. In particular, if $f$ is already ordered, then a general circuit computing $f$ can be efficiently simulated by an ordered circuit. Every ordered circuit is syntactically multilinear, as defined in Section 7.2 below. This implies that non-commutative circuits for ordered polynomials can be efficiently simulated by syntactically multilinear circuits. We do not know such a result in the commutative world: the best known transformation of a commutative circuit to a syntactically multilinear circuit increases the size by a factor of $2^{r}$ (instead of $r^{3}$ here).

The theorem is a stronger version of Theorem 1.11 which was stated in the introduction.

Theorem 7.1. Let $\Phi$ be a circuit of size $s$ computing a homogeneous polynomial $f$ of degree $r$. Then there is an ordered circuit $\Psi$ of size $O\left(r^{3} s\right)$ that computes $f^{(\text {ord })}$.

Proof. Before we prove the theorem we introduce some notation. If $g$ is a polynomial (not necessarily homogeneous) and $I=\left[j_{1}, j_{2}\right] \subseteq[r]$ is a non-empty interval, define $g^{(I)}$ as the polynomial of type $I$ defined by

$$
\operatorname{COEF}_{\alpha^{(I)}}\left(g^{(I)}\right)=\operatorname{COEF}_{\alpha}(g),
$$

where for $\alpha=\prod_{j=j_{1}}^{j_{2}} x_{i_{j}}$ we have $\alpha^{(I)}=\prod_{j=j_{1}}^{j_{2}} x_{j, i_{j}}$, and if $I=\emptyset, g^{(I)}$ is the constant term in $g$. We thus have that $f^{(\text {ord })}=f^{(I)}$ with $I=[1, r]$.

We prove the theorem by describing how to construct $\Psi$. We duplicate each gate $v$ in $\Phi$ into $O\left(r^{2}\right)$ gates in $\Psi$, which we denote by $(v, I)$ with $I \subseteq[r]$ an interval. Every $(v, I)$ will compute the polynomial $\widehat{\Phi}_{v}^{(I)}$. If $v$ is an input gate labelled by a field element, set $(v, \emptyset)=\widehat{\Phi}_{v}$ and $(v, I)=0$ for every non-empty $I$. If $v$ is an input gate labelled by a variable $x_{i}$, set $(v,[j, j])=x_{j, i}$ and $(v, I)=0$ when $I$ is not a singleton. If $v=v_{1}+v_{2}$, set $(v, I)=\left(v_{1}, I\right)+\left(v_{2}, I\right)$ for all $I$. If $v=v_{1} \times v_{2}$ and $I=[i, j]$, set

$$
(v, I)=\sum_{i-1 \leq \ell \leq j}\left(v_{1},[i, \ell]\right) \times\left(v_{2},[\ell+1, j]\right) .
$$

Associate with the gate $(v, I)$ in $\Psi$ the interval $I$. Thus, $\Psi$ is ordered. By induction, every gate $(v, I)$ computes $\widehat{\Phi}_{v}^{(I)}$, and hence $\Psi$ computes $f^{(o r d)}$. For every gate $v$ in $\Phi$, there are at most $O\left(r^{3}\right)$ edges in $\Psi$, and so the size of $\Psi$ is as claimed. 
7.2. Syntactically multilinear circuits. In this section we prove an exponential lower bound for the size of non-commutative syntactically multilinear circuits (a circuit $\Phi$ is syntactically multilinear if for every product gate $v=v_{1} \times v_{2}$ in $\Phi$, the two subcircuits $\Phi_{v_{1}}$ and $\Phi_{v_{2}}$ do not share variables). Note that an ordered circuit is automatically syntactically multilinear. By means of Theorem 7.1, a lower bound on syntactically multilinear circuits computing an ordered polynomial would imply an unconditional lower bound. However, our lower bound involves a polynomial which is not ordered.

We now define the multilinear version of central polynomials. Let $f$ be a multilinear polynomial of degree $d$. We say that $f$ is ml-central if $f$ is central as in (3.1), and for every $i \in[m]$, the polynomial $h_{i} g \bar{h}_{i}$ is multilinear; in particular, the polynomials $h_{i}, g, \bar{h}_{i}$ have distinct variables.

The following lemma describes the structure of multilinear circuits.

Lemma 7.2. Let $f$ be a homogeneous multilinear polynomial of degree $d \geq 2$. Assume that there is a syntactically multilinear circuit $\Phi$ of size s computing $f$. Then there exist $n \leq O\left(d^{3} s\right)$ and ml-central polynomials $f_{1}, \ldots, f_{n}$ such that $f=$ $f_{1}+\cdots+f_{n}$.

Proof. The proof is almost identical to Proposition 3.2

Our lower bound is based on counting monomials, similarly to Tiwari and Tompa's lower bound on the size of monotone formulas [34. The following lemma is the basic observation for the lower bound.

Lemma 7.3. Let $f$ be an ml-central polynomial of degree $k$ in $k$ variables. Then $f$ has at most $2^{-\Omega(k)} k$ ! monomials with non-zero coefficients.

Proof. Write $f$ as $f=\sum_{i \in[m]} h_{i} g \bar{h}_{i}$ with every $h_{i} g \bar{h}_{i}$ multilinear. Let $X$ be the set of variables in $f$ and $X_{0}$ the set of variables in $g$. Every monomial with a non-zero coefficient in $f$ has the form $\alpha_{1} \gamma \alpha_{2}$, where (1) $\gamma$ is a multilinear monomial of degree $d_{0}$ in variables $X_{0}$, and (2) $\alpha_{1}, \alpha_{2}$ are multilinear monomials in the variables $X \backslash X_{0}$ of degrees $d_{1}, d_{2}$, and $\alpha_{1}, \alpha_{2}$ have distinct variables. Since $d_{0}+d_{1}+d_{2}=k$, we have $\left|X_{0}\right|=d_{0}$. There are thus $d_{0}$ ! $\gamma^{\prime}$ s in $(1)$, and at most $\left(d_{1}+d_{2}\right)$ ! pairs $\alpha_{1}, \alpha_{2}$ in $(2)$. Hence $f$ contains at most

$$
d_{0} !\left(d_{1}+d_{2}\right) !=d_{0} !\left(k-d_{0}\right) !=\frac{k !}{\left(\begin{array}{c}
k \\
d_{0}
\end{array}\right)}
$$

monomials with non-zero coefficients. Since $k / 3 \leq d_{0}<2 k / 3$, this is at most $2^{-\Omega(k)} k !$.

Define the all-permutations polynomial, $\mathrm{AP}_{k}$, as a polynomial in the variables $x_{1}, \ldots, x_{k}$,

$$
\mathrm{AP}_{k}=\sum_{\sigma} x_{\sigma(1)} x_{\sigma(2)} \cdots x_{\sigma(k)},
$$

where $\sigma$ is a permutation of $[k]$. Note that $\mathrm{AP}_{k}^{(\text {ord })}$ is a polynomial in $k^{2}$ variables,

$$
\mathrm{AP}_{k}^{(\text {ord })}=\sum_{\sigma} x_{1, \sigma(1)} x_{2, \sigma(2)} \cdots x_{k, \sigma(k)} .
$$

In other words, $\mathrm{AP}_{k}^{(\text {ord })}=\mathrm{PERM}_{k}$. 
Proof of Theorem 1.12. Assume that $\mathrm{AP}_{k}$ is computed by a non-commutative syntactically multilinear circuit of size $s$. By Lemma 7.2, $\mathrm{AP}_{k}$ can be written as a sum of $O\left(k^{3} s\right)$ ml-central polynomials. By Lemma 7.3. $\mathrm{AP}_{k}$ can thus have at most $O\left(2^{-\Omega(k)} k ! k^{3} s\right)$ monomials with non-zero coefficients. However, $\mathrm{AP}_{k}$ has $k$ ! monomials.

\section{ACKNOWLEDGEMENT}

The authors would like to thank the anonymous referee, whose attentive comments helped to improve the paper.

\section{REFERENCES}

1. V. Arvind and S. Srinivasan. On the hardness of the noncommutative determinant. STOC 10', pages 677-686, 2010.

2. A. Barvinok. A simple polynomial time algorithm to approximate the permanent within a simply exponential factor. Random Structures and Algorithms 14(1), pages 29-61, 1999. MR.1662270 (2000a:68044)

3. W. Baur and V. Strassen. The complexity of partial derivatives. Theoretical Computer Science (22), pages 317-330, 1983. MR693063 (84c:68027)

4. P. Burgisser. Completeness and reduction in algebraic complexity theory. Springer-Verlag, Berlin, Heidelberg, 2000. MR.1771845 (2001g:68030)

5. S. Chien and A. Sinclair. Algebras with polynomial identities and computing the determinant. SIAM J. on Comput. 37, pages 252-266, 2007. MR2306292 (2008e:68048)

6. S. Chien, L. Rasmussen and A. Sinclair. Clifford algebras and approximating the permanent. STOC 02', pages 222-231, 2002. MR2121146

7. J. von zur Gathen. Algebraic complexity theory. Ann. Rev. Comp. Sci. (3), pages 317-347, 1988. MR1001207 (91a:68150)

8. C. Godsil and I. Gutman. On the matching polynomial of a graph. Algebraic Methods in Graph Theory, pages 241-249, 1981. MR642044 (83b:05101)

9. L. Hyafil. On the parallel evaluation of multivariate polynomials. SIAM J. Comput. 8, pages 120-123, 1979. MR529584 (80c:68031)

10. P. Hrubeš, A. Wigderson and A. Yehudayoff. An asymptotic bound on composition number of integer sums of squares formulas. To appear in Canadian Mathematical Bulletin.

11. P. Hrubeš and A. Yehudayoff. Homogeneous formulas and symmetric polynomials. Comput. Complex., to appear.

12. P. Hrubeš, A. Wigderson and A. Yehudayoff. Relationless completeness and separations. CCC 10', pages $280-290,2010$.

13. A. Hurwitz. Über die Komposition der quadratischen Formen von beliebigvielen Variabeln. Nach. Ges. der Wiss. Göttingen, pages 309-316, 1898.

14. A. Hurwitz. Über die Komposition der quadratischen Formen. Math. Ann., 88, pages 1-25, 1923. MR.1512117

15. I. M. James. On the immersion problem for real projective spaces. Bull. Amer. Math. Soc. 69, pages 231-238, 1967. MR0144355 (26:1900)

16. M. Jerrum, A. Sinclair and E. Vigoda. A polynomial-time approximation algorithm for the permanent of a matrix with nonnegative entries. J. ACM 51(4), pages 671-697, 2004. MR2147852 (2006b:15013)

17. S. Jukna. Boolean function complexity: advances and frontiers. Book in preparation.

18. N. Karmarkar, R. Karp, R. Lipton, L. Lovasz and M. Luby. A Monte-Carlo algorithm for estimating the permanent. SIAM J. Comput. 22 (2), pages 284-293, 1993. MR1207785 (94h:15004)

19. T. Kirkman. On pluquatemions, and horaoid products of sums of $n$ squares. Philos. Mag. (ser. 3), 33, pages 447-459; 494-509, 1848.

20. K. Y. Lam. Some new results on composition of quadratic forms. Invent. Math. 79 (3), pages 467-474, 1985. MR782229(86e:11027)

21. T. Y. Lam and T. Smith. On Yuzvinsky's monomial pairings. Quart. J. Math. Oxford. 44 (2), pages 215-237, 1993. MR1222375 (94f:11025) 
22. K. Mulmuley. On P vs. NP, Geometric Complexity Theory, and the Riemann Hypothesis. Technical Report, Computer Science Department, The University of Chicago, 2009.

23. N. Nisan. Lower bounds for non-commutative computation. STOC 91', pages 410-418, 1991.

24. N. Nisan and A. Wigderson. Lower bounds on arithmetic circuits via partial derivatives. Computational Complexity (6), pages 217-234, 1996. MR.1486927(99f:68107)

25. A. Pfister. Zur Darstellung definiter Funktionen als Summe von Quadraten. Invent. Math. 4, pages 229-237, 1967. MR0222043 (36:5095)

26. J. Radon. Lineare Scharen orthogonalen Matrizen. Abh. Math. Sem. Univ. Hamburg 1, pages $2-14,1922$

27. R. Raz. Multi-linear formulas for permanent and determinant are of super-polynomial size. STOC 04', pages 633-641, 2004. MR2121652 (2005j:68045)

28. R. Raz. Elusive functions and lower bounds for arithmetic circuits. STOC 08', pages 711-720, 2010. MR 2582923

29. R. Raz and A. Yehudayoff. Lower bounds and separation for constant depth multilinear circuits. CCC 08', pages 128-139, 2008. MR2513495 (2010e:68076)

30. R. Raz, A. Shpilka and A. Yehudayoff. A lower bound for the size of syntactically multilinear arithmetic circuits. SIAM J. Comput. 38 (4), pages 1624-1647, 2008. MR2465561 (2010d:68053)

31. D. B. Shapiro. Composition of quadratic forms. W. de Gruyter Verlag, 2000. MR1786291 (2002f:11046)

32. V. Strassen. Die berechnungskomplexitat von elementarsymmetrischen funktionen und von interpolationskoeffizienten. Numerische Mathematik (20), pages 238-251, 1973. MR0324947 (48:3296)

33. V. Strassen. Vermeidung von Divisionen. J. Reine Angew. Math. 264, pages 182-202, 1973. MR $0521168(58: 25128)$

34. P. Tiwari and M. Tompa. A direct version of Shamir and Snir's lower bound on monotone circuit depth. IPL, pages 243-248, 1994. MR1266720 (95c:68112)

35. L. G. Valiant. Completeness classes in algebra. STOC '79, pages 249-261, 1979. MR564634 (83e:68046)

36. S. Winograd. On the number of multiplications needed to compute certain functions. Comm. on Pure and Appl. Math. (23), pages 165-179, 1970. MR0260150 (41:4778)

37. P. Yiu. Sums of squares formulae with integer coefficients. Canad. Math. Bull., 30, pages 318-324, 1987. MR906354 (88m:11019)

38. P. Yiu. On the product of two sums of 16 squares as a sum of squares of integral bilinear forms. Quart. J. Math. Oxford. 41 (2), pages 463-500, 1990. MR1081107 (91m:11027)

39. S. Yuzvinsky. A series of monomial pairings. Linear and Multilinear Algebra 15, pages 19-119, 1984. MR740665 (85e:11028)

School of Mathematics, Institute for Advanced Study, Princeton, New Jersey 08540 Current address: Department of Computer Science, University of Calgary, Alberta T2N 1N4, Canada

E-mail address: pahrubes@math.ias.edu

School of Mathematics, Institute for Advanced Study, Princeton, New Jersey 08540

E-mail address: avi@ias.edu

School of Mathematics, Institute for Advanced Study, Princeton, New Jersey 08540 Current address: Department of Mathematics, Technion-IIT, Haifa 32000, Israel

E-mail address: amir.yehudayoff@gmail.com 